\title{
Steuergerechtigkeit nach deutschem und türkischem Verfassungsrecht ${ }^{*}$
}

\author{
Von Joachim Lang
}

\section{I. $\quad$ Einleitung}

Steuergerechtigkeit ist ein Begriff, der mit sehr verschiedenen Inhalten ausgefüllt werden kann. In einem Steuerstaat wird er häufig als Rechtfertigung für die Erhöhung von Steuerlasten mißbraucht. Politische Parteien pflegen ihn zum Nutzen der von ihnen vertretenen Interessengruppen zu gebrauchen. Schließlich kann die Steuergerechtigkeit in verschiedenen Kulturen einen verschiedenen Inhalt annehmen. So wurde es in einer christlichen Gesellschaft als gerecht empfunden, wenn der Klerus keine Steuern bezahlte, denn er diente dem Staat durch Gebete. Und nach muslimischem Recht ist eine Steuer namens dschizja gerecht, die von Nichtmuslimen zum Ausgleich dafür erhoben wird, daß die Nichtmuslime ihren Glauben beibehalten dürfen.

In einem modernen Verfassungssystem verletzen Steuerprivilegien für den Klerus und Steuern, die nur von Angehörigen bestimmter Religionen erhoben werden, den Grundsatz der Steuergerechtigkeit. Dieser Grundsatz bildet zunächst ein leeres Gefäß, das mit Grundwerten des Rechts aufgefüllt werden muß. Erst mit der Auf nahme grundlegender Rechtswerte wandelt sich das Prinzip der Steuergerechtigkeit zu einem Rechtsprinzip, das der Besteuerung konkrete Gestalt, das heißt juristisch interpretierbare Gestalt der Gerechtigkeit zu verleihen vermag.

Solche grundlegenden Rechtswerte können in den verschiedenen Gesellschaften und Kulturkreisen sehr unterschiedlich sein. Daher muß bei der juristischen Diskussion der Steuergerechtigkeit zunächst der gemeinsame Nenner einer Ordnung von Grundwerten gefunden werden. Die Besteuerung wird durch Rechtsverhältnisse gegenüber dem Staat begründet. Daher sind die Rechtsprinzipien der Besteuerung aus der Verfassung des Staates, also aus dem Verfassungsrecht, abgeleitet. Das Verfassungsrecht trägt das System des Steuerrechts. Die verfassungsrechtlich abgeleiteten Rechtsprinzipien der Besteuerung bezeichnen wir als systemtragende Prinzipien des Steuerrechts.

* Die türkische Version des zugrundeliegenden Vortrags auf dem türkischen Finanzkongress v. 13.16. Mai 1998 in Bodrum (Titel: "Vergi adaletinin anayasal temelleri") ist veröffentlicht im Jahrbuch des XIII. Türkiye maliye sempozyumu "Anayasal mali düzen".

1 Siehe Klaus Tipke, Die Steuerrechtsordnung, Band I, Köln 1993, S. 111 ff.; Tipke / Lang, Steuerrecht, 16. Aufl., Köln 1998, § 4 Rz. 70 ff. 
Die Verfassung der Republik Türkei von 1982 enthält eine Ordnung von Grundwerten, die mit denen westeuropäischer Verfassungen übereinstimmt. Der gemeinsame Ursprung dieser Ordnungen ist die französische Erklärung der Menschen- und Bürgerrechte von 1789 mit den Grundwerten der Freiheit, Gleichheit und Brüderlichkeit. Im Unterschied zu den nordamerikanisch geprägten Verfassungsordnungen, die dem Wert individueller Freiheit den höchsten Rang einräumen, ist die türkische Verfassung ebenso wie die kontinentaleuropäischen Verfassungen wesentlich auch von der Sozialbindung (toplum yararina baglilik) der Freiheitsrechte geprägt. Die türkische Republik gewährleistet nicht nur individuelle Freiheit; sie ist Sozialstaat ebenso wie Frankreich, Italien, Spanien, Deutschland und andere kontinentaleuropäische Staaten. Damit können wir eine gemeinsame Trias von Grundwerten der Freiheit, Gleichheit und der sozialen Solidarität als Basis der verfassungsrechtlichen Bestimmung von Steuergerechtigkeit feststellen.

Der Ausgangspunkt für diese verfassungsrechtliche Bestimmung der Steuergerechtigkeit ist der materielle Rechtsstaat mit dem Ziel des Staates, eine gerechte Rechtsordnung zu schaffen. ${ }^{2}$ Die Erfahrung mit dem Nationalsozialismus in Deutschland hat uns gelehrt, daß sich der Rechtsstaat nicht in formellen Kriterien der Gesetzmäßigkeit und des Rechtsschutzes erschöpfen darf. Das nationalsozialistische Gesetz ordnete zum Beispiel gegenüber den Juden höchstes Unrecht an. Deshalb muß das Recht im Rechtsstaat dadurch gesichert werden, daß den Gesetzen materielle Gerechtigkeit abverlangt wird. In einem materiellen Rechtsstaat sind Rechtsprechung und Exekutive nicht lediglich an das Gesetz, sondern an das Junktim von "Gesetz und Recht" gebunden. Das ist die Erfahrung in Deutschland, die in Art. 20 der deutschen Verfassung, im Grundgesetz (GG) von 1949 ihren Niederschlag gefunden hat. Das Junktim von "Gesetz und Recht" wird hier deshalb so deutlich herausgestellt, weil in keinem Land der Welt Steuergesetze so häufig einer verfassungsrechtlichen Überprüfung der Steuergerechtigkeit unterworfen werden wie in Deutschland. Deutsche Steuerpolitiker beklagen sich immer stärker darüber, daß sich die Gesetzgebungsgewalt von Bonn nach Karlsruhe, dem Sitz des Bundesverfassungsgerichts, verlagert habe. Allerdings ist die Vielzahl der Verfassungsbeschwerden gegen Steuergesetze der Beweis dafür, wie tief der Anspruch auf materielle Rechtsstaatlichkeit in das Bewußtsein der Bürger eingedrungen ist. Der materielle Rechtsstaat ist zur Verfassungswirklichkeit Deutschlands geworden.

Die türkische Verfassung (TV) enthält in Art. 2 ein klares Bekenntnis zum materiellen Rechtsstaat, indem die Republik Türkei als ein im Geiste der Gerechtigkeit die Menschenrechte achtender Rechtsstaat definiert wird. Dort ist auch der Sozialstaat verankert. Gemäß Art. 2 TV ist die Republik Türkei ein sozialer Rechtsstaat, dessen verfassungsrechtliche Ordnung der Grundwerte sehr große Ähnlichkeit mit der Wertordnung der deutschen Ver-

2

Grundlegend zur materiellen Rechtsstaatlichkeit im Steuerrecht Klaus Tipke, Steuerrechtsordnung (Fn. 1), S. 135 ff., und Tipke / Lang, Steuerrecht (Fn. 1), § 4 Rz. 60 ff. 
fassung aufweist. Dies macht es interessant, hier die verfassungsgerichtliche Judikatur und Lehren zu diskutieren, die in Deutschland zur verfassungsrechtlich bestimmten Steuergerechtigkeit entwickelt worden sind. Die gemeinsame Grundlage des türkischen und des deutschen Verfassungsrechts ist der materielle Rechtsstaat, dessen Steuergesetze die Grundwerte der Gleichheit, der Freiheit und der sozialen, das heißt sozialstaatlich geprägten Steuergerechtigkeit zu beachten haben.

\section{Bestimmung der Steuergerechtigkeit durch den Gleichheitssatz}

\section{Zum gemeinsamen Inhalt der Art. 10 TV; 3 GG: Gleichheit vor dem Gesetz und spezielle Diskriminierungsverbote}

\subsection{Gleichheit vor dem Gesetz (Rechtsanwendungsgleichheit) und Vereinbarkeit von Gesetzen mit dem Gleichheitssatz}

Das Bundesverfassungsgericht verankert den Grundsatz der Steuergerechtigkeit primär im Gleichheitssatz. ${ }^{3}$ Art. 3 des Grundgesetzes stimmt im wesentlichen mit Art. 10 der türkischen Verfassung überein. Auch Art. 3 Abs. 1 des Grundgesetzes statuiert die Gleichheit vor dem Gesetz, also die gleichmäßige Anwendung der Gesetze. Gleichwohl hat das Bundesverfassungsgericht schon sehr früh die Anforderung materieller Rechtsstaatlichkeit erkannt, daß das Gesetz selbst Gleichheit gewährleisten muß. Die Rechtsprechung des Bundesverfassungsgerichts betrifft hauptsächlich die Vereinbarkeit von Steuergesetzen mit dem Gleichheitssatz. Die in Art. 3 Abs. 1 unmittelbar formulierte Rechtsanwendungsgleichheit ist vom Bundesverfassungsgericht erst relativ spät zur Frage der unvollständigen Besteuerung von Zinsen thematisiert worden.

So die ständige Rechtsprechung, amtliche Sammlung (BVerfGE), Band 6, S. 70; Bd. 9, S. 9; Bd. 13 , S. 202, 298, 338; Bd. 23, S. 253; Bd. 26, S. 310; Bd. 29, S. 335; Bd. 36, S. 330, Bd. 43, S. 118; Bd. 47, S. 29; Bd. 49, S. 360; Bd. 50, S. 391; Bd. 65, S. 354; Bd. 66, S. 223. Zum Verhältnis von Gerechtigkeit und Gleichheit siehe Klaus Tipke, Steuerrechtsordnung (Fn. 1), S. 313 ff.

Urteil des Zweiten Senats vom 27.6.1991, BVerfGE Bd. 84, S. 239, zur Frage der unvollständigen Besteuerung von Zinsen. In den Leitsätzen heißt es: "Der Gleichheitssatz verlangt für das Steuerrecht, daß die Steuerpflichtigen durch ein Steuergesetz rechtlich und tatsächlich gleich belastet werden. Die Besteuerungsgleichheit hat mithin als ihre Komponenten die Gleichheit der normativen Steuerpflicht ebenso wie die Gleichheit bei deren Durchsetzung in der Steuererhebung... Der Gesetzgeber muß die Steuerehrlichkeit deshalb durch hinreichende, die steuerliche Belastungsgleichheit gewährleistende Kontrollmöglichkeiten abstützen ..." Berichterstatter dieses Urteils war Paul Kirchhof, dem eine Reihe grundlegender Entscheidungen des Bundesverfassungsgerichts zu verdanken sind. In den Gründen des Urteils (S. 260 ff.) wird der faktische Zustand, daß Privatpersonen Zinsen in Deutschland überwiegend nicht versteuern, ausführlich geschildert. Dazu auch Klaus Tipke, Die rechtliche Misere der Zinsbesteuerung, Betriebs-Berater 1989, S. 147. Inzwischen haben die Behörden der Steuerfahndung (nach § 208 Abs. 1 Nr. 1 zuständig für die Erforschung von Steuerstraftaten) bei deutschen Großbanken in zum Teil spektakulärer Weise ermittelt. 


\subsection{Die speziellen Diskriminierungsverbote in Art. 3 Abs. 3 GG und die Privilegierung von Religionsgemeinschaften in Deutschland}

a) Ebenso wie Art. 3 Abs. 3 GG enthält Art. 10 TV entsprechend dem internationalen Menschenrechtsstandard strikte Diskriminierungsverbote und daraus folgend klare Kriterien der Steuergerechtigkeit: Jedermann ist ohne Rücksicht auf Unterschiede aufgrund von Sprache, Rasse, Farbe, Geschlecht, politischer Ansicht, Weltanschauung, Religion, Bekenntnis und ähnlichem gleichzubehandeln. Die diskriminierende Besteuerung der Juden im nationalsozialistischen Unrechtsstaat ist ein evidentes Beispiel für Steuerungerechtigkeit.

Wie schon zu Beginn festgestellt, verletzen Steuerprivilegien für den Klerus und Steuern, die nur von Angehörigen bestimmter Religionen erhoben werden, in einem modernen Verfassungssystem den Grundsatz der Steuergerechtigkeit. Dies folgt aus der strikten Gleichbehandlung der Religionen, die eine moderne rechtsstaatliche Verfassung zu gebieten hat. Das laizistische Element des türkischen Rechtsstaats vertieft die Religionsneutralität der Steuergerechtigkeit. Eine Steuer für Nichtmuslime wie die dschizja wäre weder mit dem laizistischen Prinzip des türkischen Rechtsstaats (Art. 2 TV) noch mit dem Verbot religiöser Diskriminierung in Art. 10 TV zu vereinbaren.

b) Allerdings räumt das Grundgesetz den Religionsgemeinschaften, die als Körperschaften des öffentlichen Rechts anerkannt sind, einen Sonderstatus ein, der neben anderen Privilegien das Recht zur Erhebung von Steuern einschließt. Dieser Sonderstatus ergibt sich aus Art. 137 der Weimarer Reichsverfassung (WV) vom 11.8.1919. Art. 140 GG hat das in den Art. 136 bis 139, 141 WV geregelte Religionsverfassungsrecht in das Grundgesetz übernommen. Das aus der Weimarer Reichsverfassung übernommene Religionsverfassungsrecht und das Grundrecht der Religionsfreiheit (Art. 4 GG) sind als Einheit zu sehen und aufeinander abgestimmt zu interpretieren. ${ }^{5}$ Der in Art. $137 \mathrm{WV}$ normierte privilegierte Status der Religionsgemeinschaften verdeutlicht eine Sicht der Religionsfreiheit, die sich wesentlich von der laizistischen Konzeption der türkischen Verfassung unterscheidet.

Das laizistische Prinzip weist die Ausübung der Religion der freiheitsrechtlich geschützten Privatsphäre zu, in der das Individuum sein Recht auf gedankliche und emotionale Persön-

Die Situation hat sich nicht grundlegend verbessert. Viele Deutsche haben ihr privates Geldvermögen nach Luxemburg, Österreich und an andere Plätze gebracht, wo Zinsen nicht besteuert werden. Kritisch zur aktuellen Lage Klaus Tipke, Zur Steuerfahndung bei Banken und Bankkunden, Betriebs-Berater 1998, S. 241.

So die ständige Rechtsprechung des Bundesverfassungsgerichts, amtliche Sammlung (BVerfGE)

Band 19, S. 206, 216; Bd. 24, S, 236, 246; Bd. 44, S. 37, 49 f.; Bd. 53, S. 366, 406. 
lichkeit und würde frei von der Einwirkung des Staates und der Politik entfalten kann. ${ }^{6}$ Demgegenüber wird in Deutschland die öffentlich-rechtliche Privilegierung damit gerechtfertigt, daß dieser Status die Eigenständigkeit und Unabhängigkeit der Religionsgemeinschaften bekräftigt. ${ }^{7}$ Demnach gilt die Privilegierung der Religionsgemeinschaften, besonders die Berechtigung zur Erhebung von Steuern (Art. 140 GG in Verbindung mit 137 Abs. $6 \mathrm{WV}$ ), die von den staatlichen Finanzbehörden vollzogen wird, als Beitrag zur Religionsfreiheit. ${ }^{8}$

Die Privilegierung der Religionsgemeinschaften muß mit der Gleichheit der Religionsausübung vereinbart werden. Art. 3 Abs. 3 GG verbietet nämlich ausdrücklich nicht nur die Diskriminierung, sondern auch die Privilegierung bestimmter Religionen. Ebenso wie der Laizismus untersagt auch das Grundgesetz die Staatsreligion (Art. 137 Abs. 1 WV in Verbindung mit Art. 140 GG). Die Religionsgleichheit wird durch den Grundsatz der Neutralität hergestellt, nach dem der Staat die Vielfalt der religiösen und weltanschaulichen Positionen bejaht. ${ }^{9}$ Im Sinne dieser Neutralität kann nach Art. 137 Abs. 5 Satz 2 WV in Verbindung mit Art. 140 GG jede Religionsgemeinschaft den Status einer Körperschaft des öffentlichen Rechts erlangen, wenn sie durch ihre Verfassung und die Zahl ihrer Mitglieder die Gewähr der Dauer bietet. Die nach Art. 137 Abs. 5 Satz 2 WV eingerichtete Religionskörperschaft nimmt keine Staatsaufgaben wahr; sie ist vielmehr wegen des Verbots der Staatsreligion wesensmäßig vom Staat getrennt. Jedoch verleiht die Verfassung der Religionsgemeinschaft einen Korporationsstatus, welcher einerseits die Religionskörperschaft privilegiert, ihr sogar ein mit dem Staat verwirklichtes Besteuerungsrecht verleiht, der aber

Dazu insbesondere das "Kopftuch"-Urteil des türkischen Verfassungsgerichts vom 7.3.1989, deutsche Fundstelle: Europäische Grundrechte Zeitschrift 1990, S. 146. Deutsche Literatur: E.E. Hirsch, die Verfassung der Türkischen Republik vom 9. November 1982, Eine Einführung, Jahrbuch des öffentlichen Rechts der Gegenwart, Bd. 32 (1983), S. 507, 524 (Religion als Privatsache); C. Rumpf, Das Laizismusprinzip in der Rechtsordnung der RepublikTürkei, Jahrbuch des öffentlichen Rechts der Gegenwart, Bd. 36 (1987), S. 179 ff.; ders., Das türkische Verfassungssystem, Einführung mit vollständigem Verfassungstext,Wiesbaden 1996, S. $106 \mathrm{f}$. Allerdings weicht Art. 24 Abs. 3 TV insofern vom laizistischen Prinzip ab, als die Religions- und Sittenerziehung und -lehre der Aufsicht und Kontrolle des Staates unterworfen und die religiöse Kulturund Sittenlehre als Pflichtfach des Schulunterrichts angeordnet wird.

So das Bundesverfassungsgericht, BVerfGE Bd. 30, S. 415, 428.

Dazu C. Starck, in: v. Mangoldt / Klein, Das Bonner Grundgesetz, Kommentar, Art. 4 Rdbr. 79 f.; G. Robbers, Förderung der Kirchen durch den Staat, in: Handbuch des Staatskirchenrechts der Bundesrepublik Deutschland, Bd. I, Berlin 1994, S. 867, und speziell zur Kirchensteuer: $W$. Rüfner, Zur Verfassungsmäßigkeit der Kirchensteuer, Neue Juristische Wochenschrift 1971, S. 15; H. Marré, Die Kirchensteuer als Paradigma staatlicher Kirchenförderung, Kirche und Recht, 1995, S. 410.

9 Siehe A. v. Campenhausen, in: v. Mangoldt / Klein, Das Bonner Grundgesetz, Kommentar, Art. 140 Rdnr. 16 f., 18. 
andererseits auch Rechtstreue und Loyalität verlangt, die für eine dauerhafte Zusammenarbeit unerläßlich sind. ${ }^{10}$

Den muslimischen Gemeinschaften ist bisher der Status einer Körperschaft des öffentlichen Rechts mit der Begründung verweigert worden, es fehle ihnen derzeit noch eine Verfassung, die sie in den Stand setzt, ein partnerschaftliches, auf dauerhafte Kooperation angelegtes Verhältnis zum Staat zu entwickeln. ${ }^{11}$ Die Behandlung der muslimischen Gemeinschaften zeigt, daß die Sicherung der Religionsfreiheit durch einen verfassungsrechtlichen Sonderstatus der Religionsgemeinschaften ihren Preis hat: Aus laizistischer Sicht wird durch einen privilegierenden Korporationsstatus, der notwendig mit dauerhafter Kooperation zwischen Religionsgemeinschaft und Staat verknüpft ist, die Gleichheit der Religionsausübung beeinträchtigt. Hingegen wird in Deutschland ganz überwiegend die Auffassung vertreten, die Religionsgleichheit sei nicht verletzt, weil jede Religionsgemeinschaft den verfassungsrechtlichen Sonderstatus beantragen könne. ${ }^{12}$ Diese Auffassung vermag vom laizistischen Standpunkt wohl nicht zu überzeugen, weil nicht jede Religionsgemeinschaft die Bedingungen des verfassungsrechtlichen Sonderstatus erfüllen kann, ohne dadurch in ihrer religiösen Eigenart beeinträchtigt zu sein. So leistet das laizistische Modell des türkischen Rechtsstaats in bezug auf Religionsgleichheit mehr Steuergerechtigkeit als das Grundgesetz.

Dazu zuletzt grundsätzlich das Urteil des Bundesverwaltungsgerichts vom 26.6.1997, amtliche Sammlung (BVerwGE), Band 105, S. 117, das den Zeugen Jehovas die Anerkennung als Körperschaft des öffentlichen Rechts versagte, weil den Zeugen Jehovas die Teilnahme an staatlichen Wahlen nicht gestattet ist. Zu dem Korporationsstatus A. v. Campenhausen (Fn. 9), Rdnr. 145 ff.; E. Friesenhahn, Die Kirchen und Religionsgemeinschaft als Körperschaften des öffentlichen Rechts, in: Handbuch des Staatskirchenrechts der Bundesrepublik Deutschland, Bd. I, 1974, S. 545 ff.; $R$. Smend, Grundsätzliche Bemerkungen zum Korporationsstatus der Kirchen, Zeitschrift für evangelisches Kirchenrecht 1971, S. 241 ff.; H. Weber, Die Religionsgemeinschaften als Körperschaften des öffentlichen Rechts im System des Grundgesetzes, 1966, S. 46 ff.

Dazu S. Muckel, Muslimische Gemeinschaften als Körperschaften des öffentlichen Rechts, DÖV 1995, S. $311 \mathrm{ff}$. 


\section{Gleichmäßige Besteuerung nach der Leistungsfähigkeit}

\subsection{Das Leistungsfähigkeitsprinzip als Vergleichsmaßstab für die Anwendung des Gleichheitssatzes im Steuerrecht}

Während die Gestalt der Steuergerechtigkeit durch die besonderen Diskriminierungsverbote der Art. 10 TV; Art. 3 Abs. 2 GG schon deutlich sichtbar und justiziabel gemacht wird, läßt der allgemeine Gleichheitssatz dem Verfassungsjuristen einen breiten Interpretationsspielraum. Das Bundesverfassungsgericht interpretiert den allgemeinen Gleichheitssatz als Willkürverbot: Gleiches darf nicht willkürlich ungleich und Ungleiches darf nicht willkürlich gleich behandelt werden. ${ }^{13}$

Das Willkürverbot macht die Interpretation des Gleichheitssatzes zur dogmatischen Grundlage einer Rechtfertigungslehre: Es geht darum, Gleichbehandlungen und Ungleichbehandlungen zu rechtfertigen. Eine solche Rechtfertigungslehre bedarf eines Vergleichsmaßstabs, eines tertium comparationis. Erst durch einen Vergleichsmaßstab gewinnt der Gleichheitssatz in dem einzelnen Rechtsgebiet seine Funktionalität.

Nach ständiger Rechtsprechung des Bundesverfassungsgerichts ${ }^{14}$ und ganz herrschender steuerrechtswissenschaftlicher Lehre ${ }^{15}$ ist das Prinzip der Besteuerung nach der wirtschaftlichen Leistungsfähigkeit der Vergleichsmaßstab für die Anwendung des Gleichheitssatzes im Steuerrecht. Daher ist das Leistungsfähigkeitsprinzip auch das Fundamentalprinzip der im Gleichheitssatz verankerten Steuergerechtigkeit. Diese Dogmatik des Gleichheitssatzes entspricht dem internationalen menschenrechtlichen Standard. Die Besteuerung nach der Leistungsfähigkeit war schon in Art. 13 der erwähnten französischen Erklärung der Menschen- und Bürgerrechte von 1789 niedergelegt. Daher ist das Leistungsfähigkeitsprinzip in zahlreichen Verfassungen ausdrücklich festgeschrieben, so auch in Art. 73 Abs. 1 TV.

Die Weimarer Reichsverfassung von 1919 bekannte sich in Art. 134 ausdrücklich zum Leistungsfähigkeitsprinzip. Das Grundgesetz enthält keine dem Art. 135 WV entspre-

Die zahlrichen Entscheidungen sind zitiert in: Leibholz / Rinck / Hesselberger, Grundgesetz für die Bundesrepublik Deutschland, Kommentar an Hand der Rechtsprechung des Bundesverfassungsgerichts, Art. 3 Rz. 21.

BVerfGE Bd. 6, S. 55, 67 (Haushaltsbesteuerung); Bd. 8, S. 51, 68 f. (Parteispenden); Bd. 9, S. 237, 243; Bd. 13, S. 290, 297; Bd. 14, S. 34, 41; Bd. 27, S. 58, 64; Bd. 32, S. 333, 339; Bd. 36, S. 66, 72; bd. 43, S. 108, 118 (Kinderfreibetrag); Bd. 47, S. 1, 29; Bd. 55, S. 274, 302; Bd. 61, S. 319, 343 ff. (Ehegattensplitting); Bd. 66, S. 214, 233 (Unterhaltsaufwendungen); Bd. 68, S. 143, 152 f.; Bd. 82, S. 60, 86 f. (Familienexistenzminimum).

15 Umfassend insbesondere D. Birk, Das Leistungsfähigkeitsprinzip als Maßstab der Steuernormen, 1983; K. Tipke, Steuerrechtsordnung (Fn. 1), S. 469 ff.; Tipke / Lang, Steuerrecht (Fn. 1), § 4 Rz. $81 \mathrm{ff}$. 
chende Regelung. Gleichwohl wird das Prinzip der Besteuerung nach der Leistungsfähigkeit aus dem allgemeinen Gleichheitssatz so konkret abgeleitet, als ob die Besteuerung nach der Leistungsfähigkeit expressis verbis in der Verfassung geregelt wäre. Art. 73 TV und die Interpretation des allgemeinen Gleichheitssatzes im deutschen Verfassungsrecht bilden also einen gemeinsamen Ausgangspunkt für die verfassungsrechtliche Bestimmung der Steuergerechtigkeit.

\subsection{Die historische Funktion des Leistungsfähigkeitsprinzips als Progressions- und Umverteilungsprinzip}

Im Zeitalter der Aufklärung, namentlich unter dem Einfluß von Charles de Montesquieu und Jean-Jacques Rousseau ${ }^{16}$, und in der sozialistischen Bewegung des 19. Jahrhunderts hat sich das Leistungsfähigkeitsprinzip als ein Prinzip der Steuerprogression und der Umverteilung entwickelt. Diese Funktion des Leistungsfähigkeitsprinzips ist in einigen europäischen Verfassungen ausdrücklich festgehalten, so zum Beispiel in Art. 53 der italienischen Verfassung. Auch Art. 73 TV ist von dieser historischen Funktion des Leistungsfähigkeitsprinzips geprägt, indem Art. 73 Abs. 2 TV die gerechte und ausgewogene Verteilung der Steuerlast zum "sozialen Ziel" der Finanzpolitik erklärt.

Die Entwicklung des Staates zu einem Steuerstaat, der immer tiefer in die Taschen der Bürger greift, hat das Leistungsfähigkeitsprinzip als Progressions- und Umverteilungsprinzip in Mißkredit gebracht. In der Tat hat eine scharfe Steuerprogression und die staatliche Lenkung von Wirtschaft und Gesellschaft die juristischen Strukturen der Steuergesetze immer tiefergreifend zerstört. In allen Steuerstaaten wird die sozialpolitische Überfrachtung der Steuergesetze beklagt. In Deutschland ist für den gegenwärtigen Zustand der Steuergesetze der Begriff "Steuerchaos" gebräuchlich geworden. Die Lenkung durch den Staat zerstört schließlich die Umverteilung durch die Steuerprogression. Viele Bürger mit den höchsten Einkommen zahlen in Deutschland keine Einkommensteuer, weil die hohe Steuerprogression sie dazu gedrängt hat, in großem Stil die steuergesetzliche Förderung Ostdeutschlands durch Sonderabschreibungen in Anspruch zu nehmen.

Für derartige Mißstände wird das Leistungsfähigkeitsprinzip verantwortlich gemacht. Die Gegner von Progression und Umverteilung sind zugleich Gegner des Leistungsfähigkeitsprinzips. Sie fordern die Abkehr vom Leistungsfähigkeitsprinzip, in der Türkei das Streichen des Leistungsfähigkeitsprinzips aus Art. 73 TV. Es wird behauptet, daß dieses Prinzip 
ein ökonomisch rationales Steuersystem und damit den Wohlstand der Nation verhindere. ${ }^{17}$ Der schärfste Gegner des Leistungsfähigkeitsprinzips in Deutschland ist der bekannte Staatsrechtler Walter Leisner. In seinem kürzlich erschienenen Werk über die Demokratie ${ }^{18}$ geißelt Walter Leisner das Leistungsfähigkeitsprinzip als Ausdruck der Brutalität des Steuerstaates: Dem Bürger, der mehr geben könne, dürfe der Staat auch mehr nehmen. Eine solche Machtmaximierung des Staates könne kein Rechtsprinzip sein. Walter Leisner hat Recht. Doch seine Vorstellungen vom Leistungsfähigkeitsprinzip ignorieren den modernen steuerrechtswissenschaftlichen Erkenntnisstand vom Leistungsfähigkeitsprinzip.

\subsection{Das moderne Verständnis des Leistungsfähigkeitsprinzips als Prinzip der Leistungsfähigkeitsindikation und als Schutzprinzip gegen übermäßige Besteuerung}

Das moderne Verständnis des Leistungsfähigkeitsprinzips ist diametral zu der Auffassung von Walter Leisner gegen den Machtmißbrauch des Staates gerichtet. Das Leistungsfähigkeitsprinzip verdient nur dann den Namen eines Rechtsprinzips und einen Platz in der Verfassung, wenn es den Bürger gegen die Übermacht des Staates schützt und dem Steuerrecht juristische und ökonomische Vernunft verleiht. Daher entfaltet sich das Leistungsfähigkeitsprinzip besonders als Schutzprinzip gegen Übermaß der Besteuerung: Es verbietet Besteuerung dort, wo Leistungsfähigkeit nicht besteht, und es wendet sich gegen ein Übermaß von Progression und Umverteilung. Als Maßstab der Steuergleichheit führt das Leistungsfähigkeitsprinzip zu Bemessungsgrundlagen ohne Ausnahmen und dementsprechend niedrigen Steuersätzen, auch zu einem ökonomisch rationalen Steuersystem mit möglichst wenigen Fehlallokationen und schließlich zu der von Prof. Dr. Eser Karakas ${ }^{19}$ geforderten Konsumorientierung des Steuersystems. Wie in den Schriften des Verfassers ${ }^{20}$ immer wieder herausgearbeitet, vermag nur die Konsumorientierung der Besteuerung von Einkommen die gleichmäßige Austeilung der Steuerlasten nach der wirtschaftlichen Lei-

So wie auch viele andere Ökonomen Prof. Dr. Eser Karakas in seinem Vortrag (S. 9 ff.) auf dem türkischen Finanzkongress v. 13.-16. Mai 1998 in Bodrum (XIII. Türkiye Maliye Sempozyumu "Anayasal Mai Düzen").

W. Leisner, Demokratie. Betrachtungen zur Entwicklung einer gefährdeten Staatsform, Berlin 1998, S. 342 ff.

Siehe E. Karakas (Fn. 17), S. 9 ff.

20 Siehe zuletzt die 16. Auflage von Tipke / Lang (Fn. 1), § 4 Rz. 110 ff., sowie J. Lang, Konsumorientierung - eine Herausforderung für die Steuergesetzgebung?, in: Smekal / Sendlhofer / Winner (Hrsg.), Einkommen versus Konsum, Ansatzpunkte zur Steuerreformdiskussion, Heidelberg 1999, S. $143 \mathrm{ff}$. 
stungsfähigkeit zu optimieren, wie noch dargelegt werden wird. ${ }^{21}$ Dieses moderne Verständnis des Leistungsfähigkeitsprinzips wird wie folgt spezifiziert:

a) Zunächst setzt die juristische Dogmatisierung des Leistungsfähigkeitsprinzips eine operable Normenlehre voraus: Die Steuerrechtswissenschaft unterscheidet zwischen Fiskalzwecknormen, die dem Zweck dienen, den Finanzbedarf des Staates zu decken, und Sozialzwecknormen, mit denen der Staat lenkend und umverteilend in die ökonomischen Verhältnisse eingreift. ${ }^{22}$ Im Rahmen dieser Lehre wird das Leistungsfähigkeitsprinzip als Rechtsprinzip für Fiskalzwecknormen entfaltet, das für die gleichmäßige Austeilung der Steuerlasten zu sorgen hat. Demgegenüber eignet sich das Leistungsfähigkeitsprinzip als Maßstab der Steuergleichheit nicht für die Rechtfertigung von Sozialzwecknormen. Vielmehr erzeugen Sozialzwecknormen Steuerungleichheit, die einer Rechtfertigung bedarf. Es muß die Abweichung der Sozialzwecknorm vom Leistungsfähigkeitsprinzip gerechtfertigt werden. $^{23}$

Das gilt besonders für die Steuerprogression, die dem Bereich der Sozialzwecknormen angehört und im Sozialstaatsprinzip begründet ist. ${ }^{24}$ Wir beurteilen die Steuerprogression als Umverteilungsnorm, die von den Beziehern höherer Einkommen mehr abschöpft, als dies der Gleichheitssatz gebietet, denn das Leistungsfähigkeitsprinzip führt entgegen der traditionellen Auffassung nicht zur Progression, sondern zur Proportion, weil die Fähigkeit, Steuern zu zahlen, nicht schneller wächst als das Einkommen. ${ }^{25}$ Daher weicht die Progressionsnorm vom Leistungsfähigkeitsprinzip ab, was durch das Sozialstaatsprinzip gerechtfertigt sein muß. ${ }^{26}$

Schließlich scheitert die juristische Verifikation des Leistungsfähigkeitsprinzips im Bereich von Sozialzwecknormen auch daran, daß das Recht auf Fragen nach der "richtigen" Progression, nach dem "richtigen" Ausgleich von Arm und Reich oder nach der "richtigen" Lenkung der Wirtschaft durch Steuervergünstigungen und Sonderbelastungen wie zum

Siehe unten II 2.4.1.2.

Siehe insbesondere D. Birk, Leistungsfähigkeitsprinzip (Fn. 14), S. 67 ff., $153 \mathrm{ff}$., $194 \mathrm{ff}$., $232 \mathrm{ff}$; K. Tipke, Steuerrechtsordnung (Fn. 1), S. 119 ff.; Tipke / Lang, Steuerrecht (Fn. 1), § 4 Rz. 20 ff., 81 ff., 124 ff.; K. Vogel, Die Abschichtung von Rechtsfolgen im Steuerrecht, Lastenausteilungs-, Lenkungs- und Vereinfachungsnormen und die ihnen zuzurechnenden Steuerfolgen: ein Beitrag zur Methodenlehre des Steuerrechts, Steuer und Wirtschaft 1997, S. 97.

Siehe Tipke / Lang, Steuerrecht (Fn. 1), § 4 Rz. 124 ff.

Dazu unten IV.

So K. Tipke, Steuerrechtsordnung (Fn. 1), S. 412. Siehe auch Tipke / Lang, Steuerrecht (Fn. 1), $\S 4$ Rz. 197, sowie M. Jachmann, Leistungsfähigkeitsprinzip und Umverteilung, Steuer und Wirtschaft 1998, S. 293.

Dazu unten IV. 
Beispiel Umweltsteuern keine verbindliche Auskunft zu geben vermag. Diese Fragen sind Sache der Politik, im schlechteren Falle Sache von Ideologien, die Wirtschaft und Gesellschaft of tmals mehr schaden als nützen.

b) Auf der Suche nach rechtswissenschaftlich rational begründbaren Steuersystemen von Fiskalzwecknormen wendet sich die Steuerrechtswissenschaft nicht der Gestaltung von Steuersätzen zu, die von den Vorgaben der Politik und des Finanzbedarfs abhängig sind. Ihr Interesse gilt vielmehr den Bemessungsgrundlagen, den Indikatoren steuerlicher Leistungsfähigkeit. Die rechtswissenschaftlich und auch wirtschaftswissenschaftlich interessanten Grundfragen lauten: Mit welchen Bemessungsgrundlagen wird die wirtschaftliche Leistungsfähigkeit am besten erfaßt? Und wenn diese Fragen beantwortet sind: Wie sind diese Bemessungsgrundlagen nach dem Leistungsfähigkeitsprinzip optimal definiert? Die letztere Frage führt die verfassungsrechtliche Bestimmung der Steuergerechtigkeit nach dem Leistungsfähigkeitsprinzip auf die Ebene konkreter, praktischer Steuerfragen. So hat zum Beispiel der Bundesfinanzhof ${ }^{27}$ handelsrechtliche Bilanzierungswahlrechte in der Steuerbilanz nicht zugelassen, weil es dem Leistungsfähigkeitsprinzip widerspreche, wenn der Steuerpflichtige seinen zu versteuernden Gewinn beliebig gestalten könne. Hieran zeigt sich, daß das Leistungsfähigkeitsprinzip im Bereich der die Leistungsfähigkeit messenden Normen rechtsdogmatisch bis hin zur Lösung von Einzelfällen operationalisiert werden kann.

Ebenso wie das türkische Verfassungsgericht ${ }^{28}$ unterscheidet auch die deutsche Steuerrechtswissenschaft drei Indikatoren steuerlicher Leistungsfähigkeit: das Einkommen, das Vermögen und den Konsum. ${ }^{29}$ Die juristische Steuersystemforschung befaßt sich hauptsächlich mit der Indikation steuerlicher Leistungsfähigkeit. ${ }^{30}$ Dabei ist allerdings in interdisziplinärer Kooperation mit den Wirtschaftswissenschaften die optimale Ausgestaltung von Bemessungsgrundlagen zu klären, zum Beispiel zu untersuchen, ob eine konsumorientierte Besteuerung von Einkommen auch aus juristischer Sicht ${ }^{31}$ die optimale Form der Besteuerung darstellt, wie es die optimal taxation theories behaupten.

Großer Senat vom 3.2.1969, Bundessteuerblatt, Jahrgang 1969, Teil II, S. 291. Weitere Nachweise: Tipke / Lang, Steuerrecht (Fn. 1), § 9 Rz. 327.

Urteile 80/27 vom 6.7.1995, 4/28 vom 6.7.1995 und 85/32 vom 13.7.1995.

Dazu mit weiteren Nachweisen Tipke / Lang, Steuerrecht (Fn. 1), § 4 Rz. 95 ff.

Dazu umfassend K. Tipke, Steuerrechtsordnung (Fn. 1), Bd. II. Vgl. auch J. Lang, Die Bemessungsgrundlage der Einkommensteuer. Rechtssystematische Grundlagen steuerlicher Leistungsfähigkeit im deutschen Einkommensteuerrecht, Köln 1988.

31 Siehe J. Lang, Entwurf eines Steuergesetzbuchs, Schriftenreihe des Bundesministeriums der Finanzen, Heft 49, Bonn 1993. In diesem im Auftrag des Bundesfinanzministeriums für die Beratung mittel- und osteuropäischer Länder verfaßten Entwurf hat der Verfasser die ökonomischen Modelle einer konsumorientierten Besteuerung von Einkommen in Gesetzes normen umgesetzt. 
c) Der bereits erwähnten Ungleichbehandlung durch Sozialzwecknormen ${ }^{32}$ wirkt das Leistungsfähigkeitsprinzip entgegen: Es entspricht dem Leistungsfähigkeitsprinzip, die in allen Steuerstaaten beklagte sozialpolitische Überfrachtung des Steuerrechts abzubauen, besonders die Bemessung von Steuern auf die Leistungsfähigkeitsindikation zurückzuschneiden und Steuervergünstigungen konsequent zu streichen. ${ }^{33}$ Kurzum: Es entspricht dem Leistungsfähigkeitsprinzip, wenn der Staat darauf verzichtet, mit Steuergesetzen Sozialpolitik zu betreiben.

Ein von Sozialzwecknormen entlastetes Steuerrecht hat der Verfasser in seinem "Entwurf eines Steuergesetzbuchs" ${ }^{\prime 34}$ ausgearbeitet. Wir haben ein solches Steuerrecht inzwischen in Kroatien verwirklicht, wo es mit großem Erfolg praktiziert wird. Dort haben wir nachgewiesen, daß der Verzicht auf steuergesetzliche Lenkung mehr soziale Gerechtigkeit und wirtschaftliche Blüte erzeugt, denn mit Steuervergünstigungen verstehen die Reichen am besten umzugehen. Die Staatsbürokratie ist auch nicht fähig, Investitionen richtig zu bewerten, so daß Steuersubventionen die sog. Fehlallokationen bewirken und häufig wirtschaftsschädlich sind.

d) Schließlich entfaltet das Leistungsfähigkeitsprinzip gegenüber Sozialzwecknormen seine im Gleichheitssatz begründete Schutzfunktion: Je schärfer der Staat Progression und Umverteilung ausgestaltet, desto stärker wird das Bedürfnis, die vom Maßstab des Leistungsfähigkeitsprinzips abweichende Ungleichverteilung der Steuerlasten vor dem Gleichheitssatz zu rechtfertigen. Im weiteren schützt das Leistungsfähigkeitsprinzip gegen die regressive Wirkung von Lenkungssteuern. Die gegenwärtige Bundesregierung hat eine ökologische Steuerreform beschlossen, die durch indirekte Ökosteuern den Energieverbrauch schrittweise stark verteuern will. ${ }^{35}$ Die Lenkungswirkungen solcher Energiesteuern treffen die sozial schwächsten Schichten am stärksten. Sie haben weder die Mittel, die höheren Energiepreise zu bezahlen, noch haben sie die Mittel für Energiesparmaßnahmen. So geht in Deutschland das Gerücht um, daß sich die Reichen auf eine drastische Erhöhung der Mineralölsteuer freuen würden, weil dann endlich mehr Platz auf den Autobahnen für ihre

Siehe oben S. 523.

Siehe oben S. 521.

Zitiert in Fn. 31.

Koalitionsvereinbarung zwischen der Sozialdemokratischen Partei Deutschlands (SPD) und der Partei Bündnis 90/Die Grünen von 20.10.1998, III 3. Die ökologische Steuerreform erstreckt sich auf folgende Energieträger: Mineralöl (Benzin), Heizöl, Gas und Strom. Die Koalitionsvereinbarung folgt damit Vorschlägen der EG-Kommission und des sozialdemokratisch orientierten Deutschen Instituts für Wirtschaftsforschung in Berlin. Dazu H.-W. Arndt, Rechtsfragen einer deutschen $\mathrm{CO}_{2} /$ Energiesteuer, entwickelt am Beispiel des DIW-Vorschlages, Afrankfurt 1995; $S$. Bach, Wirtschaftliche Auswirkungen und rechtlich- institutionelle Aspekte einer ökologischen Steuerreform, Steuer und Wirtschaft 1995, S. 264; Tipke / Lang, Steuerrecht (Fn. 1), \& 2 Rz. 57 (mit weiteren Nachweisen). 
schnellen Luxuslimousinen ist. Das Lenkungsziel der Umweltsteuer kollidiert scharf mit dem Leistungsfähigkeitsprinzip. Eine effiziente Umweltsteuer birgt notwendig soziale Härten, die von den bisherigen Vorschlägen zu einer Energiesteuer hingenommen werden. Solchen Fehlentwicklungen der Steuergerechtigkeit muß das Leistungsfähigkeitsprinzip entgegengesetzt werden. ${ }^{36}$

\subsection{Besteuerung von Einkommen, Vermögen und Konsum nach dem Leistungsfähigkeitsprinzip}

\subsubsection{Besteuerung von Einkommen}

Die traditionelle Lehre und Rechtsprechung weisen dem Einkommen die beste Qualifikation als Leistungsfähigkeitsindikator zu. Deshalb ist die Rechtsdogmatik des Leistungsfähigkeitsprinzips im Einkommensteuerrecht am weitesten fortgeschritten.

\subsubsection{Dualistisches Konzept des zu versteuernden Einkommens}

Nicht nur in Deutschland, sondern auch in vielen anderen Ländern wird die steuerliche Leistungsfähigkeit im Einkommensteuerrecht dualistisch gemessen: Es wird zunächst eine Summe verschiedener Einkunftsarten ermittelt, bei denen Erwerbsaufwendungen abgezogen werden. Diese Summe von Gewinnen und Verlusten (Summe der Einkünfte) wird sodann verringert um persönliche Abzüge, die den notwendigen Konsum des Steuerpflichtigen berücksichtigen. Die internationale Formel des zu versteuernden Einkommens lautet: Adjusted Gross Income minus Personal Deductions. Die Summe der Einkünfte (Adjusted Gross Income) erfaßt das Einkommen im wirtschaftlichen Sinne als Indikator für objektive wirtschaftliche Leistungsfahigkeit. Die persönlichen Abzüge (Personal Deductions) berücksichtigen individuellen Konsum des Steuerpflichtigen und dienen daher als Indikator für subjektive wirtschaftliche Leistungsfähigkeit. Dieses dualistische Konzept des zu versteuernden Einkommens mit einem Erwerbsfaktor und einem Konsumfaktor entspricht der finanzwissenschaftlichen Tradition. ${ }^{37}$

a) Summe der Einkünfte: Das deutsche Einkommensteuergesetz erfaßt das Einkommen im wirtschaftlichen Sinne in einer Summe von sieben Einkunftsarten ( $22 \mathrm{Abs} .1$ bis $3 \mathrm{EStG}$ ).

Dazu näher J. Lang, Verwirklichung von Umweltschutzzwecken im Steuerrecht, Deutsche Steuerjuristische Gesellschaft, Band 15 (1993), S. 115, S. 126 f., S. 157 f. Siehe auch F.R. Balmes, Verfassungsmäßigkeit und rechtliche Systematisierung von Umweltsteuern, Lohmar / Köln 1997, S. 181 ff. (Sozialverträglichkeit von Umweltsteuern).

In Deutschland geht es auf Adolph Wagner zurück (siehe J. Lang, Die Bemessungsgrundlage der Einkommensteuer [Fn. 30], S. 34 f.). 
aa) Auf dieser ersten Stufe des dualistischen Konzepts soll nach dem Leistungsfähigkeitsprinzip das Totalitätsprinzip verwirklicht werden. ${ }^{38}$ Nach diesem Prinzip hat die Einkommensteuer das gesamte Einkommen zu erfassen und gleichmäßig zu besteuern. Dem Leistungsfähigkeitsprinzip entspricht die Gesamteinkommensteuer und nicht die Schedulensteuer, die einzelne Einkunftsarten verschieden besteuert. Sowohl die unvollständige als auch die verschiedene Besteuerung der Einkunftsarten widerspricht dem Leistungsfähigkeitsprinzip. Leider ist das die Realität der meisten Einkommensteuergesetze. So verfolgen die meisten Einkommensteuerreformen das Ziel, die Unterschiede der Erfassung von Einkünften, vor allem die Steuerbefreiung bestimmter Einkünfte und andere Sozialzwecknormen zu beseitigen, um die Steuersätze absenken zu können. Hieran zeigt sich deutlich das bereits oben ${ }^{39}$ dargelegte moderne, nicht auf Progression angelegte Verständnis des Leistungsfähigkeitsprinzips.

bb) Im weiteren wird die Besteuerung der Einkünfte durch das objektive Nettoprinzip ${ }^{40}$ geprägt: Die Leistungsfähigkeit kann nicht nur nach den Einnahmen oder Erträgen gemessen werden. Vielmehr ist die richtige Meßgröße der Unterschiedsbetrag zwischen Einnahmen / Erträgen und Aufwendungen, die durch die Erwerbstätigkeit veranlaßt sind. ${ }^{41}$ Das Leistungsfähigkeitsprinzip gebietet also in Gestalt des objektiven Nettoprinzips die Ab-

Zum Totalitätsprinzip siehe J. Lang, Die Bemessungsgrundlage der Einkommensteuer (Fn. 30), S. $167 \mathrm{ff}$. Mit der Verwirklichung des Totalitätsprinzips befassen sich die verschiedenen Einkommenstheorien: Das umfassendste Konzept bietet die Reinvermögenszugangstheorie (net accretion theory - Haig / Simons / Schanz). In Deutschland ist die Markteinkommenstheorie (market income theory) entwickelt worden, die nur das erwirtschaftete Einkommen erfaßt. Nicht erfaßt sind Erbschaften und Schenkungen (sie unterliegen der Erbschaft- und Schenkungsteuer), Unterhaltszuwendungen, Wertschöpf ungen in der Privatsphäre (z.B. die Dienstleistungen einer Hausfrau) sowie Einkünfte aus einer Liebhaberei, die meistens Verluste sind. Die Markteinkommenstheorie beschränkt den Einkommensbegriff auf das juristisch erfaßbare Erwerbseinkommen und ermöglicht eine juristisch konsequentere Umsetzung des Totalitätsprinzips als die für die juristische Praxis zu weit gefaßte Reinvermögenszugangstheorie. Zur Markteinkommenstheorie ausführlich J. Lang, Bemessungsgrundlage der Einkommensteuer (Fn. 30), S. 229 ff.; K. Tipke, Steuerrechtsordnung (Fn. 1), Band II, S. 566 ff.; R. Wittmann, Das Markteinkommen - einfachgesetzlicher Strukturbegriff und verfassungsdirigierter Anknüpfungspunkt der Einkommensteuer?, Augsburg 1992. Siehe auch Doralt / Ruppe, Grundriß des österreichischen Steuerrechts, Band I, 6. Aufl., Wien 1998, S. 17/18; Blumenstein / Locher, System des Steuerrechts, 4. Aufl., Zürich 1992, S. 142.

Siehe S. 522 ff., S. 524 f.

Zum Nettoprinzip ausführlich J. Lang, Bemessungsgrundlage der Einkommensteuer (Fn. 30), S. 60 ff., S. 183 ff.; K. Tipke, Steuerrechtsordnung (Fn. 1), Band II, S. 591 ff.; Tipke / Lang, Steuerrecht (Fn. 1), § 9 Rz. 54 f. (dort Literaturübersicht in Fn. 31). Der Deutsche Juristentag, Sitzungsbericht N, München 1988, S. 214, hat proklamiert: "Das Nettoprinzip gehọrt zu den identitätskonstituierenden Merkmalen der Einkommensteuer. Als solches steht es nicht zur Disposition des Gesetzgebers."

41

Ausführlich zum Begriff der Erwerbsaufwendungen Tipke / Lang, Steuerrecht (Fn. 1), § 9 Rz. $205 \mathrm{ff}$. 
ziehbarkeit von Erwerbsaufwendungen und den Abzug von Verlusten, entweder mit positiven Einkünften derselben Periode (Verlustausgleich) oder durch Verlustvortrag und Verlustrücktrag. Der interperiodische Abzug von Verlusten ist keine Steuersubvention, sondern eine das objektive Nettoprinzip und damit das Leistungsfähigkeitsprinzip verwirklichende Fiskalzwecknorm.

b) Persönliche Abzüge: Auf der zweiten Stufe des dualistischen Konzepts ist sodann der steuerlich nicht belastbare (indisponible) Teil des Einkommens auszuscheiden. Dies geschieht durch die persönlichen Abzüge, die wir wegen der Zuordnung zur privaten Konsumsphäre auch Privatabzüge nennen.

Das deutsche Einkommensteuergesetz enthält ebenso wie andere Einkommensteuergesetze eine Vielfalt von persönlichen Abzügen, die verschiedene Konsumaufwendungen zum Steuerabzug zulassen. ${ }^{42}$ Derartige Konglomerate sind historisch willkürlich gewachsen und entsprechen daher nicht den Anforderungen einer juristischen Ordnung. Somit stellt sich im internationalen Rechtsvergleich die Frage, was nach dem Leistungsfähigkeitsprinzip abziehbar sein soll. Hierzu hat die deutsche Rechtswissenschaft das subjektive oder private Nettoprinzip entwickelt. ${ }^{43}$ Nach diesem Prinzip ist der Teil des Einkommens, der für den existenznotwendigen Lebensbedarf benötigt wird, durch den Abzug der unvermeidbaren Konsumausgaben von der "Summe der Einkünfte" aus der Bemessungsgrundlage auszuscheiden. Diese Lehre vom "indisponiblen Einkommen" ist durch grundlegende Entscheidungen des Bundesverfassungsgerichts von $1990^{44}$ und $1992^{45}$ anerkannt worden.

Nach $\S 2$ Abs. 4 und 5 EstG Sonderausgaben, außergewöhnliche Belastungen, der Kinderfreibetrag und der Haushaltsfreibetrag. Als Sonderausgaben sind zum Beispiel nach $\S 10$ EstG absetzbar Unterhaltsleistungen an den geschiedenen Ehegatten, Beiträge zu bestimmten Versicherungen (z.B. Kranken-, Unfall-, Haftpflichtversicherungen), Kirchensteuer, Steuerberatungskosten, Kosten der Berufsausbildung, Aufwendungen für eine Hausgehilfin. Außergewöhnliche Belastungen ( $\S \S 33 ; 33 a ; 33 b ; 33 c$ EstG) sind z.B. Krankheitskosten, Kosten einer Ehescheidung, Aufwendungen für die Wiederbeschaffung von Kleidung und Hausrat nach Brand, Naturkatastrophen, Kriegen, bestimmte Unterhaltsaufwendungen, Pauschalen für Körperbehinderte. Zu der Vielfalt der privaten Abzüge siehe Tipke / Lang, Steuerrecht (Fn. 1), § 9 Rz. 700 ff.

Grundlegend K. Tipke, Steuerrecht - Chaos, Konglomerat oder System?, Steuer und Wirtschaft 1971, S. 2 ff., S. 16 f. Siehe im weiteren K. Tipke, Steuerrechtsordnung (Fn. 1), Band II, S. 672 ff.; Tipke / Lang, Steuerrecht (Fn. 1), § 9 Rz. 68 ff. (mit aktueller Literaturübersicht). Der Deutsche Juristentag (Fn. 40), S. 214, hat proklamiert: "Der Einkommensteuer unterliegt nur der Teil des Erwerbseinkommens, der für den Steuerpflichtigen disponibel ist. Die unvermeidbaren Aufwendungen für die eigene Existenzsicherung und den Unterhalt der Familienangehörigen müssen deshalb von der Besteuerung freigestellt sein ..."

Beschluß vom 25.5.1990, amtliche Sammlung (BVerfGE), Band 82, S. $60 \mathrm{ff}$.

Beschluß vom 25.9.1992, amtliche Sammlung (BVerfGE), Band 87, S. 153 ff. 
Die Lehre vom "indisponiblen Einkommen" vollzieht eine gemeinsame Aufgabe von Steuerrecht und Sozialrecht, welche die türkische Verfassung ebenso wie die deutsche Verfassung gebietet. Art. 5 TV verpflichtet den Staat dazu, die für die materielle und ideelle Existenz des Menschen notwendigen Bedingungen zu schaffen. Die Bundesrepublik Deutschland ist ebenso wie die Republik Türkei ein Sozialstaat (siehe unten IV.). Daher hat der Bürger einen verfassungsrechtlichen Anspruch auf die Gewährleistung des "sozialkulturellen Existenzminimums". Das Sozialhilferecht hat das "sozial-kulturelle Existenzminimum" durch staatliche Zuwendungen zu sichern, wenn der Bürger kein Einkommen hat. Hat der Bürger Einkommen, so muß das Einkommen in Höhe der Sozialhilfe steuerfrei sein. Die Rechtsordnung hat nämlich die verfassungsrechtlich bestimmte Gerechtigkeit widerspruchsfrei zu verwirklichen. Diesen verfassungsrechtlich verbindlichen Zusammenhang zwischen Einkommensteuerrecht und Sozialhilferecht hat das Bundesverfassungsgericht $1992^{46}$ grundlegend bestätigt.

\subsubsection{Konsumorientierung der Besteuerung von Einkommen}

Nach dem dualistischen Konzept des zu versteuernden Einkommens enthält die Einkommensteuer einen gewichtigen Konsumfaktor, nämlich die Abzugsfähigkeit unvermeidbarer Konsumausgaben. Diese Konsumorientierung der Besteuerung von Einkommen ist dem Charakter einer progressiven Einkommensteuer adäquat, der opfertheoretisch gerechtfertigt wird: Nach der klassischen Opfertheorie ist die Relation zwischen der Höhe des Einkommens und dem Umfang der Bedürfnisbefriedigung durch fallenden Grenznutzen gekennzeichnet. Zusätzliche Einkommensteile werden für die Bedürfnisbefriedigung immer unwichtiger, so daß sie höherer Steuerbelastung zugänglich sind. ${ }^{47}$ Danach ist das Einkommen in Höhe des existenznotwendigen Lebensbedarfs steuerfrei zu stellen. In Höhe des Luxuskonsums kann das Einkommen besonders hoch besteuert werden.

Diese opfertheoretische Rechtfertigung des progressiven Einkommensteuertarifs knüpft an das Potential der Bedürfnisbefriedigung, also an eine Konsumleistungsfähigkeit an. Damit stellt sich die Frage, ob nicht opfertheoretisch folgerichtig bereits die "Summe der Einkünfte" konsumorientiert zu definieren ist, und zwar als eine Summe der für den gegenwärtigen Konsum verfügbaren Einkünfte, während gesparte und investierte Einkünfte erst später in dem Zeitpunkt zu besteuern sind, in dem sie dem Potential der Bedürfnisbefriedigung zugeführt werden.

46

47

Beschluß vom 25.9.1992 (Fn. 45).

Dazu ausführlich K. Schmidt, Die Steuerprogression, Tübingen 1960, S. $16 \mathrm{ff}$., S. $51 \mathrm{ff}$;; $\mathrm{H}$. Haller, Die Steuern, Grundlinien eines rationalen Systems öffentlicher Abgaben, 3. Aufl., Tübingen 1981, S. 76 ff. Siehe auch F.K. Mann, Steuerpolitische Ideale (Fn. 16); F. Neumark, Grundsätze gerechter und ökonomisch rationaler Steuerpolitik, Tübingen 1970, S. 122 f., S. 176 f. 
Damit wird ein umstrittenes Thema angeschnitten, nämlich die von Prof. Dr. Eser Karakas geforderte Konsumorientierung des Steuersystems. Herkömmlicherweise wird der Konsum durch indirekte Steuern besteuert. Hier geht es um die Konsumorientierung der direkten Steuern. Das klassische Modell der direkten Steuer auf den Konsum ist die Ausgabensteuer. ${ }^{48}$ Dieses Modell ist noch 1989 in Heidelberg ernsthaft diskutiert worden. ${ }^{49}$ Die Untauglichkeit der Ausgabensteuer ist mittlerweile allgemein erkannt worden: Privatkonsum ist technisch schwer zu erfassen. Die internationale Verflechtung der Steuersysteme durch Doppelbesteuerungsabkommen macht auch die Einführung von Ausgabensteuern unmöglich, weil alle Vertragsstaaten die direkte Besteuerung radikal reformieren müßten. ${ }^{50}$ Daher sucht man seit 1989 nach Methoden der Konsumorientierung im herkömmlichen Normensystem direkter Steuern. ${ }^{51}$ Diese Methoden definieren Einkünfte konsumorientiert. In der juristischen Praxis sind folgende Methoden anzutreffen

a) Sparbereinigung der Einkünfte (cash flow): Einzahlungen in einen Sparfonds (Pensionsfonds, Lebensversicherung etc.) sind abziehbar. Die Auszahlungen aus dem Sparfonds sind zu versteuern. Diese Form der Besteuerung ist in vielen Ländern schon bei der steuerlichen Behandlung gesetzlicher Rentenversicherungen anzutreffen. Auch Beamte sind cashflow-besteuert: Die Vorsorge des Staates für die Pension des Beamten werden nicht als Einkünfte des Beamten erfaßt. Vielmehr wird erst die Auszahlung der Pension an den

Siehe I. Fisher, Income in Theory and Income Taxation in Practice, Econometrica 5, Wisconsin 1937, S. 1 ff.; N. Kaldor, An Expenditure Tax, London 1955; R. Peffekoven, Persönliche allgemeine Ausgabensteuer, in: Handbuch der Finanzwissenschaft, Band II, 3. Aufl., Tübingen 1980, S. $418 \mathrm{ff}$.

Siehe M. Rose (ed.), Heidelberg Congress on Taxing Consumption, Proceedings of the International Congress on Taxing Consumption, held at Heidelberg, June 28-30, 1989, Springer-Verlag, Heidelberg 1990.

50

Siehe Heidelberg Congress (Fn. 49), S. 453 ff. (Taxing Consumption from an International Point of View, with contributions by P.N. Musgrave, N. Andel, K. Vogel, G. Krause-Junk, B. Gense); Joseph / Vollrath, Anwendung des Internationalen Rechts bei Einführung einer Ausgabensteuer, München 1991.

51

Siehe bereits $J$. Lang, Taxing Consumption from a Legislative Point of View, Heidelberg Congress (Fn. 49), S. 273 ff., und J. Lang, Entwurf eines Steuergesetzbuchs (Fn. 31): Cash-FlowMethode bei der Einkommensteuer und Methode der Zinsbereinigung bei der Unternehmensteuer. Grundlegende Literatur: David F. Bradford, Untangling the Income Tax, Harvard University Press 1986; McLure / Zodrow, Heidelberg Congress (Fn. 49), S. 335 ff.; M. Rose, Reform der Besteuerung des Sparens und der Kapitaleinkommen, Betriebs-Berater Beilage Nr. 5/1992; F.W. Wagner, Die zeitliche Erfassung steuerlicher Leistungsfähigkeit, in: Hax / Kern / Schröder (Hrsg.), Zeitaspekte in betriebswirtschaftlicher Theorie und Praxis, Stuttgart 1989, S. 261, S. 270 ff.; E. Wenger, Gleichmäßigkeit der Besteuerung von Arbeits- und Vermögenseinkünften, Finanzarchiv Band 41 (1983), S. 207. Aktuelle Literatur: M. Rose (Hrsg.), Standpunkte zur aktuellen Steuerreform, Heidelberg 1997; M. Rose, Konsumorientierung des Steuersystems - Steuersysteme der Zukunft, Berlin 1998, S. 247 ff.; Smekal / Sendlhofer / Winnwe (Hrsg.), Einkommen versus Konsum (Fn. 20). Weitere Quellen: Tipke / Lang, Steuerrecht (Fn. 1), § 4 Rz. 110 ff. 
Beamten der Einkommensteuer unterworfen. Neuerdings bezeichnet man die Cash-flowBesteuerung in Deutschland als "nachgelagerte" Besteuerung.

b) Zinsbereinigung der Einkünfte: Danach werden Einkünfte nur besteuert, soweit sie die marktübliche Normalverzinsung des eingesetzten Kapitals überschreiten. Diese Form der Konsumorientierung wird seit 1995 mit großem Erfolg in Kroatien praktiziert. ${ }^{52}$

Beide Methoden (Sparbereinigung und Zinsbereinigung der Einkünfte) sind für den Steuerzahler ökonomisch gleichwertig. Sie führen rechnerisch zum gleichen Ergebnis der Steuerbelastung, wie das folgende Schaubild 1 (Entwicklung eines Sparguthabens bei einem Steuersatz von $30 \%$ ) zeigt. Für den Fiskus erweist sich allerdings die Methode der Zinsbereinigung als vorteilhafter, weil dem Fiskus die Steuern früher und permanenter zufließen

Schaubild 1: Entwicklung eines Sparguthabens bei einem Steuersatz von $30 \%$

\begin{tabular}{|c|c|c|c|c|c|c|c|}
\hline $\begin{array}{c}\text { Alter } \\
\text { des } \\
\text { Sparers }\end{array}$ & $\begin{array}{c}\text { Sparguthaben } \\
\text { ohne Steuem } \\
\text { in DMc }\end{array}$ & $\begin{array}{l}\text { Sparguthaben } \\
\text { bei } \\
\text { zinsbereinigter } \\
\text { Einkommen- } \\
\text { steuer } \\
\text { in DM }\end{array}$ & $\begin{array}{l}\text { Steuer- } \\
\text { last }\end{array}$ & $\begin{array}{l}\text { Sparguthaben } \\
\text { bei } \\
\text { sparbereinigter } \\
\text { Einkommen- } \\
\text { steuer } \\
\text { in DM }\end{array}$ & $\begin{array}{l}\text { Steuer- } \\
\text { last }\end{array}$ & $\begin{array}{c}\text { Sparguthaben } \\
\text { bei } \\
\text { traditioneller } \\
\text { Einkommen- } \\
\text { steuer } \\
\text { in DM }\end{array}$ & $\begin{array}{c}\begin{array}{c}\text { Steuer- } \\
\text { last }\end{array} \\
\text { in \% }\end{array}$ \\
\hline $\begin{array}{l}25 \\
26 \\
27 \\
28 \\
29 \\
30 \\
\bullet \\
\bullet \\
60 \\
61 \\
62 \\
63 \\
64 \\
65\end{array}$ & $\begin{array}{c}10.000 \\
10.600 \\
11.236 \\
11.910 \\
12.625 \\
13.382 \\
\bullet \\
76.861 \\
81.473 \\
86.361 \\
91.543 \\
97.035 \\
102.857\end{array}$ & $\begin{array}{c}7.000 \\
7.420 \\
7.865 \\
8.337 \\
8.837 \\
9368 \\
\bullet \\
53.803 \\
57.031 \\
60.453 \\
64.080 \\
67.925 \\
72.000\end{array}$ & $\begin{array}{c}30 \\
30 \\
30 \\
30 \\
30 \\
30 \\
\bullet \\
\bullet \\
30 \\
30 \\
30 \\
30 \\
30\end{array}$ & $\begin{array}{c}10.000 \\
10.600 \\
11.236 \\
11.910 \\
12.625 \\
13.382 \\
\bullet \\
76.861 \\
81.473 \\
86.361 \\
91.543 \\
97.035 \\
102.857 \\
-30.857\end{array}$ & $\begin{array}{l}0 \\
0 \\
0 \\
0 \\
0 \\
0 \\
: \\
0 \\
0 \\
0 \\
0 \\
0 \\
0 \\
0\end{array}$ & $\begin{array}{c}7.000 \\
7.294 \\
7.600 \\
7.920 \\
8.252 \\
8.599 \\
\vdots \\
29.544 \\
30.785 \\
32.078 \\
33.425 \\
34.829 \\
36.292\end{array}$ & $\begin{array}{c}30,00 \\
31,19 \\
32,36 \\
33,51 \\
34,63 \\
35,74 \\
\vdots \\
61,56 \\
62,21 \\
62,86 \\
63,49 \\
64,11 \\
64,72\end{array}$ \\
\hline & $\begin{array}{l}102.857 \mathrm{DM} \\
\text { Konsum im } \\
\text { Alter ohne } \\
\text { Steuem }\end{array}$ & $\begin{array}{l}72.000 \mathrm{DM} \\
\text { Konsum im } \\
\text { Alter nach } \\
\text { zinsbereinigter } \\
\text { Einkommen- } \\
\text { steuer }\end{array}$ & $\begin{array}{c}30 \% \\
\text { Finale } \\
\text { Steuer- } \\
\text { last }\end{array}$ & $\begin{array}{l}72.000 \mathrm{DM} \\
\text { Konsum im } \\
\text { Alter nach } \\
\text { sparbereinigter } \\
\text { Einkommen- } \\
\text { steuer }\end{array}$ & $\begin{array}{c}30 \% \\
\text { Finale } \\
\text { Steuer- } \\
\text { last }\end{array}$ & $\begin{array}{c}36.292 \mathrm{DM} \\
\text { Konsum im } \\
\text { Alter nach } \\
\text { traditioneller } \\
\text { Einkommen- } \\
\text { steuer }\end{array}$ & $\begin{array}{c}64,72 \% \\
\text { Finale } \\
\text { Steuer- } \\
\text { last }\end{array}$ \\
\hline
\end{tabular}

Dazu Greß / Rose / Wiswesser, Marktorientierte Einkommensteuer, Das neue kroatische System einer konsum- und damit marktorientierten Besteuerung des persönlichen Einkommens, München 1998. 
als im Falle der Sparbereinigung. Die Methode der Zinsbereinigung genießt allerdings geringere politische Akzeptanz, weil sich die Steuerbefreiung von Zinsen bei periodischer Betrachtung als Steuerprivileg der Kapitalisten darstellt.

Entgegen einem verbreiteten Irrtum privilegieren die Methoden der Konsumorientierung das Sparen und Investieren nicht. Vielmehr beseitigen sie die Diskriminierung des Zukunftskonsums durch die traditionelle Einkommensteuer, die von dem Sparen soviel abschöpft, daß der sofortige Konsum des Einkommens einen höheren Wert hat als der durch Sparen finanzierte Zukunftskonsum. Sie schaffen überperiodische Steuergleichheit oder intertemporale Neutralität sowie Inflationsneutralität. Sie sorgen zudem dafür, daß die Steuerlast durch den progressiven Einkommensteuertarif nicht zusätzlich zur sozialstaatlich gerechtfertigten vertikalen Ungleichheit auch noch horizontal ungleich verteilt wird.

Beispiel: Ein großer Tennisstar erwirtschaftet in wenigen Jahren sein Lebenseinkommen. Infolge der Progression wird das hohe Einkommen zunächst vertikal ungleich, das heißt mit einem höheren Steuersatz belastet als niedrigere Einkommen. Sodann erhöht die Konzentration des Lebenseinkommens auf wenige Jahre und Steuerperioden nochmals progressiv die Steuerlast, so daß der Tennisstar ökonomisch geradezu gezwungen wird, seinen Wohnsitz an Orten zu begründen, wo Einkommen niedrig oder gar nicht besteuert sind. Die Cash-flow-Besteuerung ermöglicht die Verteilung des sehr hohen Periodeneinkommens auf die Lebenszeit und erzeugt damit horizontale, das heißt in der Bemessungsgrundlage begründete Steuergleichheit.

Die Konsumorientierung der Einkünfte verfolgt also das Ziel einer überperiodisch gleichmäßigen Besteuerung nach der Leistungsfähigkeit. Das Schaubild 1 zeigt, daß die überperiodische Steuerbelastung des Sparens ohne Konsumorientierung bereits bei einem Proportionalsteuersatz von $30 \%$ in einem Zeitraum von 40 Jahren auf $64,72 \%$, also auf mehr als das Doppelte ansteigt. Demgegenüber sorgen die Methoden der Konsumorientierung dafür, daß die Steuerbelastung interperiodisch konstant bleibt. Diese Methoden entkräften auch die Kritik der Steuerprogression, sie verfälsche die Lohn- und Preisgerechtigkeit und bestrafe den Tüchtigen ${ }^{53}$, da die progressive Ungleichverteilung der Steuerlasten auf der horizontalen Ebene der Bemessungsgrundlagen beseitigt wird: Durch Sparen kann sich nämlich der Tüchtige dem aktuellen Zugriff der Steuerprogression entziehen.

53

Grundlegend die Kritik von F.A. Hayek, Die Ungerechtigkeit der Steuerprogression, in: Schweizer Monatshefte, 32. Jahrgang, 1950, und von K. Schmidt, Die Steuerprogression (Fn. 47), S. 44 ff. 


\subsubsection{Besteuerung von Vermögen}

Die deutsche Steuerrechtswissenschaft vertritt überwiegend die Auffassung, daß das Vermögen als solches nicht geeignet sei, steuerliche Leistungsfähigkeit zu indizieren. ${ }^{54}$ Gegenstand des Streits ist hauptsächlich die Vermögensteuer. Sie wird als zusätzliche Belastung des sogenannten fundierten Einkommens mit dem Argument gerechtfertigt, Vermögenseinkünfte seien leichter zu erzielen als Arbeitseinkünfte. Diese sogenannte Fundustheorie ${ }^{55}$ verletzt den Grundsatz der gleichmäßigen Besteuerung von Einkommen. Im Hinblick auf die leicht verdienten Einkünfte von Film- und Sportstars ist eine unterschiedliche Belastung von Arbeits- und Vermögenseinkünften nicht mehr zu rechtfertigen.

Die Besteuerung ertraglosen Vermögens verletzt auch das Prinzip, nur die reale Ist-Leistungsfähigkeit zu belasten. In den Fällen ertraglosen Vermögens und besonders während Verlustperioden eines Unternehmens fehlt häufig die Liquidität zur Steuerzahlung. Wenn aber der Steuerzahler zur Aufnahme eines Bankkredits oder zur Vermögensliquidation gezwungen wird, büßt der Steuerzahler Vermögen ein, das den zu zahlenden Steuerbetrag weit übersteigt. Dadurch wird das verfassungsrechtliche Übermaßverbot verletzt. Schließlich ist es kaum möglich, Bewertungsgleichheit zwischen dem Geldvermögen und dem Sachvermögen herzustellen. Besonders die Werte von Kunstgegenständen und größeren Vermögenseinheiten wie z.B. Unternehmen sind of tmals sehr schwer zu ermitteln. Daher sind nach unserer Auffassung nur die realisierten Vermögensmehrungen in Gestalt von tatsächlich erwirtschaftetem Einkommen geeignet, gleichmäßig steuerliche Zahlungsfähigkeit zu messen.

Das Bundesverfassungsgericht ${ }^{56}$ hat allerdings 1995 die Fundustheorie nicht verworfen und die verfassungsrechtliche Zulässigkeit der Vermögensteuer grundsätzlich bejaht. Jedoch hat es entschieden, daß das normale, familiäre Gebrauchsvermögen freizustellen ist $^{57}$ und daß die Vermögensteuer zusammen mit anderen Steuerbelastungen die Hälfte des Sollertrages nicht übersteigen dürfe (sog. Halbteilungsgrundsatz). ${ }^{58}$ Diese Auflagen des

Grundlegend $K$. Tipke, Steuerrechtsordnung (Fn. 1), Band II, S. 775 ff. Zur aktuellen Diskussion siehe D. Birk (Hrsg.), Besteuerung von Erbschaft und Vermögen, Deutsche Steuerjuristische Gesellschaft, Band 22, Köln 1999.

Ausführlich zu dieser Fundustheorie K. Tipke, Steuerrechtsordnung (Fn. 1), Band II, S. 775 ff.

Beschluß zur Vermögensteuer vom 22.6.1995, amtliche Sammlung (BVerfGE), Band 93, S. 121, S. $139 \mathrm{f}$.

57

Beschluß zur Vermögensteuer vom 22.6.1995 (Fn. 56), S. 138, S. 140 f., und Beschluß zur Erbschaftsteuer vom 22.6.1995, BVerfGE, Band 93, S. 174. Das Bundesverfassungsgericht bemißt das normale, familiäre Gebrauchsvermögen nach den Werten durchschnittlicher Einfamilienhäuser (BVerfGE Band 93, S. 174).

Leitsatz 3 des Beschlusses zur Vermögensteuer vom 22.6.1995 (Fn. 56), S. 121. 
Bundesverfassungsgerichts hat der Gesetzgeber bisher nicht erfüllt, so daß die Vermögensteuer gegenwärtig nicht erhoben werden darf.

Die gegenwärtige Bundesregierung möchte die Vermögensteuer wieder zum Leben erwecken. ${ }^{59}$ In den parteipolitischen Programmen empfehlen die Sozialdemokraten eine Vermögensteuer von $1 \%$ auf Privatvermögen ab einem Freibetrag für das familiäre Gebrauchsvermögen von 1.000.000 DM. ${ }^{60}$ Demgegenüber fordern die Grünen eine Vermögensteuer von $1 \%$ auf das private und das unternehmerische Vermögen und wollen einen Freibetrag für das Gebrauchsvermögen von nur 400.000 DM anerkennen. Der Halbteilungsgrundsatz verlangt allerdings eine Senkung des Spitzensatzes der Einkommensteuer von derzeit $53 \%$ auf unter $50 \%$. Dazu hat die Koalition beschlossen, den Spitzensatz der Einkommensteuer ab 1.1.2002 auf 48,5\% abzusenken. ${ }^{61}$ Allerdings wird jetzt von Sachverständigen befürchtet, daß das Aufkommen der Vermögensteuer wegen der vom Bundesverfassungsgericht festgelegten Bedingungen in einem zu großen Mißverhältnis zum Verwaltungsauf wand steht, der besonders durch die permanente Bewertung des Grundbesitzes verursacht wird. So treten zu den zahlreichen verfassungsrechtlichen, steuersystematischen und wirtschaftspolitischen Einwänden gegen die Vermögensteuer noch die fiskalische Unergiebigkeit und administrative Nachteile hinzu, so daß gegenwärtig die Wiederbelebung der nach unserer Auffassung mit dem Leistungsfähigkeitsprinzip unvereinbaren Vermögensteuer nicht geplant ist.

\subsubsection{Besteuerung des Konsums}

Die gegenwärtigen Steuersysteme besteuern den Konsum im wesentlichen durch indirekte Steuern (Umsatzsteuer und spezielle Verbrauchsteuern). Da diese Art von Steuern auf die persönliche Leistungsfähigkeit der mit der Steuer belasteten Bürger keine Rücksicht nimmt, ist die Auffassung verbreitet, daß sich das Leistungsfähigkeitsprinzip nur durch Steuern auf das Einkommen entfalten könne. Hingegen hat Klaus Tipke einen alle Steuern umfassenden

Die Koalitionsvereinbarung vom 20.10.1998 (Fn. 35), III 2, hat beschlossen: Die neue Bundesregierung wird eine Sachverständigenkommission einberufen, die die Grundlage für eine wirtschafts- und steuerpolitisch sinnvolle Vermögensbesteuerung schaffen soll."

60

Das Erbschaftsteuerrecht ist der Rechtsprechung des Bundesverfassungsgerichts bereits angepaßt worden (siehe Tipke / Lang, Steuerrecht [Fn. 1], § 13 Rz. 101, 145). In § 16 des Erbschaft- und Schenkungsteuergesetzes (ErbStG) in der Fassung vom 27.2.1997, Bundesgesetzblatt, Jahrgang 1997, Teil I, S. 378, wird das normale Gebrauchsvermögen mit einem Freibetrag von 600.000 DM für den Ehegattenerwerb und mit einem Freibetrag von 400.000 DM für Kindererwerbe berücksichtigt. Hinterläßt demnach ein tödlich verunglückter Vater seiner Frau und seinen drei Kindern ein Vermögen von 1.800.000 DM, so fällt nach § 16 ErbStG keine Erbschaftsteuer an.

61

Koalitionsvereinbarung vom 20.10.1998 (Fn. 35), III 1. 
Ansatz entwickelt. ${ }^{62}$ Nach unserer Auffassung ist das Leistungsfähigkeitsprinzip ein universales Rechtsprinzip der Besteuerung. ${ }^{63}$ Dieses Prinzip ist wohl bei den einzelnen Steuerarten mit unterschiedlicher Intensität verwirklicht. Jedoch darf keine Steuer vom Anspruch des Bürgers auf Steuergerechtigkeit ausgenommen werden. Daher ist das Leistungsfähigkeitsprinzip bei allen Steuerarten zu beachten.

So gebietet das Leistungsfähigkeitsprinzip, das "sozial-kulturelle Existenzminimum" auch bei den indirekten Steuern freizustellen. ${ }^{64}$ In seinem "Entwurf eines Steuergesetzbuchs" empfiehlt der Verfasser, die Umsatzsteuerbelastung des existenznotwendigen Lebensbedarfs konkordant zur einkommensteuerrechtlichen Regelung zu vergüten. ${ }^{65}$ Eine derartige Regelung enthielt bereits das deutsche Umsatzsteuergesetz von 1919; sie wurde schon 1920 wieder abgeschafft. ${ }^{66}$ Derartige Steuervergütungen lassen sich nach der Überzeugung des Verfassers durchaus verwaltungspraktikabel gestalten. Jedenfalls hat die verfassungsrechtliche Gewährleistung des Existenzminimums in den Sozialstaaten Türkei und Deutschland einen so hohen Rang, daß beliebige Gründe der Steuerpraktikabilität nicht ausreichen dürfen, um den steuergerechten Schutz des Existenzminimums zu verhindern. Das Bedürfnis nach einer Vergütung der das Existenzminimum belastenden Steuer wird besonders bei der bereits oben ${ }^{67}$ angesprochenen Einführung indirekter Ökosteuern unabweisbar.

\subsubsection{Zusammenfassung}

Zusammenfassend ist festzustellen, daß das Leistungsfähigkeitsprinzip in keinem Teil der Steuerrechtsordnung ausgeschaltet werden darf. Maßgrößen steuerlicher Leistungsfähigkeit sind die dynamischen Stromgrößen des Einkommens und des Konsums, während sich das Vermögen, besonders das ertraglose Vermögen, nicht als Indikator steuerlicher Leistungsfähigkeit eignet. In einem ganzheitlich am Leistungsfähigkeitsprinzip ausgerichteten Steuersystem bilden die Maßgrößen "Einkommen" und "Konsum" keinen Gegensatz, sondern nur verschiedene Ansatzpunkte der Besteuerung. Im Mittelpunkt steht der Konsumfonds des Bürgers, aus dem seine steuerliche Leistungsfähigkeit geschöpft werden kann, entweder

62

63

64

65

66

67

So besonders Band II des in Fn. 1 zitierten dreibändigen Werks "Die Steuerrechtsordnung".

Siehe auch Tipke/Lang, Steuerrecht [Fn. 1], § 4 Rz. 81 ff., Rz. 85.

Siehe mit weiteren Nachweisen K. Tipke, Steuerrechtsordnung (Fn. 1), Band II, S. 922 ff.

J. Lang, Entwurf eines Steuergesetzbuchs (Fn. 31), § 473, Begründung: Rz. 705 ff., Rz. 731.

$\S 14$ des deutschen Umsatzsteuergesetzes vom 24.12.1919 sah eine Vergütung der geschätzten Umsatzsteuerbelastung unbemittelter Individuen vor. Nach J. Popitz, Allgemeine Verbrauchsteuer, in: Handbuch der Finanzwissenschaft, Band II, Tübingen 1929, S. 187, wurde sie abgeschafft, weil sie ein "verletzendes Almosen" darstelle. F. Neumark, Grundsätze (Fn. 47), S. 83 (dort Fn. 10), vermutet Gründe der Praktikabilität.

Siehe S. $525 \mathrm{f}$. 
durch Besteuerung des Einkommens als Zufluß in den Konsumfonds oder durch Besteuerung des Konsums als Abfluß aus dem Konsumfonds. Demnach ist die Konsumleistungsfähigkeit der Gegenstand eines alle Steuerarten umspannenden Leistungsfähigkeitsprinzips, das sowohl für die gerechte Besteuerung der Einkommen als auch für eine möglichst gerechte Besteuerung der Einkommensverwendungen zu sorgen hat.

\subsection{Der verfassungsrechtliche Schutz von Ehe und Familie im Steuerrecht}

Die Rechtsprechung des Bundesverfassungsgerichts hat bisher das Leistungsfähigkeitsprinzip im Bereich der Familienbesteuerung am striktesten verwirklicht, weil Art. 6 Abs. 1 GG Ehe und Familie unter den besonderen Schutz der staatlichen Ordnung stellt. ${ }^{68}$ In ähnlicher Weise wie das Grundgesetz schützt auch die türkische Verfassung die Familie in Art. 41, so daß die Rechtsprechung des Bundesverfassungsgerichts zur Besteuerung der Familie von hohem rechtsvergleichenden Interesse ist.

Das Bundesverfassungsgericht leitet aus Art. 6 Abs. 1 GG sowohl ein Förderungsgebot als auch ein Diskriminierungsverbot ab. ${ }^{69}$ Während das Förderungsgebot hauptsächlich für das Sozialrecht Bedeutung hat, entfaltet sich Art. 6 Abs. 1 GG im Steuerrecht vor allem als ein die Anwendung des Gleichheitssatzes verschärfendes Diskriminierungsverbot. Hierzu hat das Bundesverfassungsgericht in einer Reihe sehr grundlegender Entscheidungen ${ }^{70}$ folgende drei durch die Steuerrechtswissenschaft entwickelte Regeln der Familiensteuergerechtigkeit bestätigt:

a) Individualbesteuerung: Bis 1957 wurden in Deutschland Ehegatten als eine Steuereinheit behandelt. Die Einkommen der Ehegatten wurden zusammengerechnet. Dadurch wurden

Wichtigste Entscheidungen: Beschluß vom 17.1.1957, amtliche Sammlung (BVerfGE), Band 6, S. 55 ff. (Haushaltsbesteuerung von Ehegatten); Urteil vom 3.11.1982, BVerfGE, Band 61, S. 319 ff. (Ehegattensplitting); Beschluß vom 22.2.1984, BVerfGE, Band 66, S. 214 ff. (realitätsgerechte Berücksichtigung von Unterhaltsleistungen); Beschluß vom 4.10.1984, BVerfGE, Band 67, S. 290 ff. (realitätsgerechte Berücksichtigung von Unterhaltsleistungen); Beschluß vom 17.10.1984, B VerfGE, Band 68, S. 143 ff. (Besteuerung unverheirateter Eltern); Beschluß vom 29.5.1990, BVerfGE, Band 82, S. 60 ff. (Familienexistenzminimum); Beschluß vom 12.6.1990, BVerfGE, Band 82, S. 198 ff. (Kinderfreibetrag). Zur Rechtsprechung des Bundesverfassungsgerichts pars pro toto: J. Lang, Steuer und Wirtschaft 1983, S. 103 ff.; ders., Steuer und Wirtschaft 1990, S. 331 ff.; W. Lingemann, Das rechtliche Konzept der Familienbesteuerung, Berlin 1994. M. Pechstein, Familiengerechtigkeit als Gestaltungsaufgabe für die staatliche Ordnung, Baden-Baden 1994; H.-J. Pezzer, Verfassungsrechtliche Perspektiven der Familienbesteuerung, in: Festschrift für W. Zeidler, Berlin / New york 1987, S. 757 ff.; K. Tipke, Steuerrechtsordnung (Fn. 1), Band II, S. 672 ff.; K. Vogel, Steuer und Wirtschaft 1984, S. $197 \mathrm{ff}$.

Insbesondere die in Fn. 68 zitierten Entscheidungen. 
Ehegatten progressiv höher belastet als unverheiratete Steuerzahler. In einer grundlegenden Entscheidung von $1957^{71}$ wurde die Zusammenrechnung der Einkommen in Gestalt der sog. Haushaltsbesteuerung als verfassungswidrig verworfen. Das Bundesverfassungsgericht konkretisierte die gleichmäßige Besteuerung nach der Leistungsfähigkeit durch den Grundsatz der Individualbesteuerung: Zuordnungssubjekt steuerlicher Leistungsfähigkeit und damit auch des progressiven Einkommensteuertarifs sei die einzelne natürliche Person. Der Gesetzgeber ersetzte die Haushaltsbesteuerung durch ein Ehegattensplitting ( $\S 26 ; 32 \mathrm{a}$ Abs. 5 EStG). 1982 bekräftigte das Bundesverfassungsgericht das Ehegattensplitting als eine sachgerechte Fiskalzwecknorm. Das Ehegattensplitting sei keine Steuervergünstigung; vielmehr berücksichtige es sachgerecht die Einkommensverteilung innerhalb der ehelichen Erwerbs- und Verbrauchsgemeinschaft. ${ }^{72}$

b) Steuerfreiheit des Familien-Existenzminimums: Die bereits erwähnte Lehre vom indisponiblen Einkommen bestätigte das Bundesverfassungsgericht 1990 in zwei grundlegenden Entscheidungen zum Kindergeld und zum Kinderfreibetrag. ${ }^{73}$ Der existenznotwendige Lebensbedarf der Kinder sei entweder durch einen Kinderfreibetrag oder durch ein Kindergeld steuerfrei zu stellen.

Nach diesen Entscheidungen hat der Gesetzgeber ab 1996 den sog. Familienleistungsausgleich in den $\S \S 31 ; 32 ; 62-78$ EStG neu geregelt: Die Eltern erhalten zunächst als Steuervergütung ein monatliches Kindergeld von jeweils 220 DM für das erste und zweite Kind, 300 DM für das dritte Kind und 350 DM für die weiteren Kinder. Bewirkt ein Kinderfreibetrag von jährlich 6.912 DM (sozialhilferechtliches Kinder-Existenzminimum ab 1997) eine höhere Steuerentlastung als die Beträge des Kindergeldes, so wird bei der Veranlagung zur Einkommensteuer von Amts wegen der Kinderf reibetrag angesetzt.

c) Realitätsgerechte Berücksichtigung von Unterhaltsverpflichtungen: Schließlich leitete das Bundesverfassungsgericht aus dem Leistungsfähigkeitsprinzip die Regel ab, daß Unterhaltsverpflichtungen realitätsgerecht zu berücksichtigen seien, weil Unterhaltsverpflichtungen die steuerliche Leistungsfähigkeit des Verpflichteten mindern würden. ${ }^{74}$ Wie allerdings eine solche Regel auszusehen hat, hat das Bundesverfassungsgericht offengelassen.

71

72

73

74

Beschluß vom 17.1.1957 (Fn. 68)

Urteil vom 3.11.1982 (Fn. 68), S. 345 f., im Anschluß an J. Lang, Bemessungsgrundlage der Einkommensteuer (Fn. 30), S. $620 \mathrm{ff}$.

Beschlüsse vom 29.5.1990 (Fn. 68) und vom 12.6.1990 (Fn. 68).

So insbesondere die Beschlüsse vom 22.2.1984 (Fn. 68) und vom 4.10.1984 (Fn. 68). 
Der Deutsche Juristentag hat $1988^{75}$ und $1994^{76}$ ein Familien-Realsplitting ${ }^{77}$ empfohlen, das an die gesetzlichen Verpflichtungen des Zivilrechts anknüpft: Der Leistende hat einen Steuerabzug in Höhe der gesetzlichen Unterhaltsverpflichtung, und dementsprechend hat der Empfänger Unterhaltsbezüge zu versteuern. Sodann ist bei allen Familienmitgliedern das Existenzminimum in sozialhilferechtlicher Höhe abzusetzen. Dadurch wird die Familiensteuergerechtigkeit nach dem Grundsatz der Einheit der Rechtsordnung in vollen Einklang mit dem Zivilrecht und dem Sozialrecht gebracht. Das folgende Schaubild 2 zeigt das Schema des Familien-Realsplittings an dem Beispiel einer vierköpfigen Familie.

Schaubild 2: Schema eines Familienrealsplittings

\begin{tabular}{|c|c|c|c|c|}
\hline & Vater & Mutter & Tochter & Sohn \\
\hline Markteinkommen & ME & & & \\
\hline Eherechtlicher Transfer & $-T_{e}$ & $+T_{e}$ & & \\
\hline Unterhaltsrechtlicher Transfer & $-T_{u}$ & $-T_{u}$ & $+T_{u}$ & $+T_{u}$ \\
\hline $\begin{array}{l}\text { Sozialhilferechtliches } \\
\text { Existenzminimum } \\
\text { a) staatliche Zuwendungen } \\
\text { (z.B. Kindergeld) }\end{array}$ & $+Z_{k}$ & & & \\
\hline $\begin{array}{l}\text { b) sozialhilferechtlicher } \\
\text { Lebensbedarf } \\
\text { (Erwachsene, Kinder) }\end{array}$ & $-L_{e}$ & $-L_{e}$ & $-L_{k}$ & $-L_{k}$ \\
\hline
\end{tabular}

Sitzungsbericht N zum 57. Deutschen Juristentag, München 1988, S. 213 (Unterhaltsbezüge sind im tatbestandlichen Rahmen der Einkunftsarten steuerpflichtig) und S. 216 (realitätsgerechte Berücksichtigung bedeutet Abzug in Höhe der gesetzlichen Unterhaltspflichten des bürgerlichen Rechts von der Bemessungsgrundlage).

Sitzungsbericht O zum 60. Deutschen Juristentag, München 1994, S. 92 (das Ehegattensplitting ist in eine Form der Familienbesteuerung umzugestalten, welche das Familienexistenzminimum in Übereinstimmung mit dem Sozialhilferecht steuerfrei stellt und die familienrechtlich vorgegebene Einkommensverteilung in Ehe und Familie nachvollzieht).

$\mathrm{Zu}$ diesem Familien-Realsplitting J. Lang, Bemessungsgrundlage der Einkommensteuer (Fn. 30), S. 650 ff.; ders., Referat auf dem 60. Deutschen Juristentag, Sitzungsbericht O, S. 61 ff.; ders., Entwurf eines Steuergesetzbuchs (Fn. 31), § 134, Begründung: Rz. 566, Rz. 620; R. Lehner, Einkommensteuerrecht und Sozialhilferecht, Tübingen 1993, S. 166 ff.; M. Lingemann, Familienbesteuerung (Fn. 68), S. 156 ff.; M. Moderegger, Der verfassungsrechtliche Familienschutz und das System des Einkommensteuerrechts, Baden-Baden 1991, S. 164, S. 176 ff. 


\begin{tabular}{|lcccc|}
\hline $\begin{array}{l}\text { Zu versteuerndes } \\
\text { Einkommen }\end{array}$ & $\underline{\text { Vater }}$ & $\underline{\text { Mutter }}$ & $\underline{\text { Tochter }}$ & $\underline{\text { Sohn }}$ \\
& $M E-T_{e}-T_{u}+Z_{k}-L_{e}$ & $T_{e}-T_{u}-L_{e}$ & $T_{u}-L_{k}$ & $T_{u}-L_{k}$ \\
\hline
\end{tabular}

\section{Bestimmung der Steuergerechtigkeit durch die Freiheitsrechte}

Während es wohl international üblich ist, die Steuergerechtigkeit wesentlich durch den Gleichheitssatz und das Leistungsfähigkeitsprinzip zu bestimmen, entwickelte der Richter des Bundesverfassungsgerichts Paul Kirchhof die Steuergerechtigkeit freiheitsrechtlich. Die Steuer sei eine Institution der Freiheit und der privatnützigen Eigentumsordnung. ${ }^{78}$ Im Unterschied zu einem Staat, der seinen Finanzbedarf durch staatliche Zwangswirtschaft befriedige, sei der freiheitlich organisierte Staat darauf angewiesen, sich durch Teilhabe am Erfolg privaten Wirtschaftens zu finanzieren. Davon ausgehend bindet Paul Kirchhof die Steuer in die Sozialpflichtigkeit des Eigentums (Art. 14 Abs. 1 Satz 1 GG) ein.

Die Lehre von Paul Kirchhof verbindet klassische äquivalenztheoretische Vorstellungen, wie sie bereits Adam Smith und Lorenz von Stein formuliert haben, mit moderner Grundrechtsdogmatik und gewinnt dadurch höchst originelle Interpretationsansätze, die mir für die türkische Verfassungsjurisprudenz als besonders interessant erscheinen, weil Art. 12 Abs. 2 TV die soziale Verantwortung des Individuums deutlicher und umfassender mit den Grundfreiheiten verknüpft als die deutsche Grundrechtsdogmatik. Die Kirchhof'schen Schlußfolgerungen fügen sich zu einem sehr konkreten Bild der Steuergerechtigkeit zusammen und stellen nach meiner Auffassung auch eine sinnvolle Ergänzung der am Leistungsfähigkeitsprinzip orientierten Gleichheitsdogmatik dar. ${ }^{79}$

So rechtfertigt Paul Kirchhof die Besteuerung von Einkommen damit, daß Einkommen nicht nur durch die Anstrengung des Marktanbieters, sondern auch durch eine entsprechende Nachfrage entsteht. Es seien also die von der Gemeinschaft erzeugten und gesicherten Marktbedingungen, die ursächlich für die Entstehung von Einkommen seien. Daher

Grundlegend $P$. Kirchhof, Besteuerung und Eigentum, Veröffentlichungen der Vereinigung der Deutschen Staatsrechtslehrer, Band 39 (1981), S. 213 ff.; ders., Staatliche Einnahmen, in: Isensee / Kirchhoff (Hrsg.), Handbuch des Staatsrechts der Bundesrepublik Deutschland, Band IV, Heidelberg 1990, §§ 88 Rn. 92 ff.; ders., Die verfassungsrechtliche Rechtfertigung der Steuern, in: Kirchhof / Birk / Lehner (Hrsg.), Steuern im Verfassungsstaat, München 1996, S. 27 ff. Kritisch zur Rechtfertigungstheorie von P. Kirchhof äußert sich K. Tipke, Steuerrechtsordnung (Fn. 1), Band II, S. 528 ff.

Dazu näher J. Lang, Über das Ethische der Steuertheorie von Klaus Tipke, in: Festschrift für Klaus Tipke, Köln 1995, S. 3, S. 18 ff.; Tipke / Lang, Steuerrecht (Fn. 1), § 4 Rz. 226 f. 
sei es gerecht, wenn die Gemeinschaft am Erfolg des Wirtschaftens beteiligt sei. Die indirekte Besteuerung bereitet Paul Kirchhof weniger Schwierigkeiten als den Dogmatikern des Leistungsfähigkeitsprinzips: Die indirekte Steuer erfasse "nur den anonymen Leistungsaustausch am Markt", belaste "in diesem Tatbestand den marktabhängigen Konsum" und wirke "in der Individualbesteuerung regressiv" ${ }^{80}$. Der regressive Typus einer Steuer kann auf der Grundlage einer Marktteilhabedogmatik offenbar leichter hingenommen werden als vom Standpunkt des Leistungsfähigkeitsprinzips aus. Praktisch bedeutet dies, daß das von Paul Kirchhof entworfene freiheitsrechtliche Konzept einen höheren Anteil indirekter Steuern verträgt als ein am Leistungsfähigkeitsprinzip orientiertes Konzept.

Gleichwohl plädiert Paul Kirchhof im Zusammenwirken von progressiver Einkommensteuer und regressiv wirkenden indirekten Steuern für eine stärkere Belastung desjenigen, der Einkommen "durch Nutzung des Marktes mit wachsendem Erwerbserfolg" erwirtschaftet. ${ }^{81}$ Der "wachsende Erwerbserfolg" bildet die Grundlage für die Rechtfertigung der Einkommensteuerprogression, die allerdings durch den erwähnten, aus Art. 14 Abs. 2 Satz 2 GG abgeleiteten Halbteilungsgrundsatz begrenzt wird. Paul Kirchhof denkt ganzheitlich, betrachtet demnach die Gesamtsteuerbelastung.

Der Einfluß von Paul Kirchhof auf die Rechtsprechung seines Senats ist höchst eindrucksvoll. In der Entscheidung des Bundesverfassungsgerichts von 1992 wird die Steuerfreiheit des Existenzminimums freiheitsrechtlich auf das in den Art. 12 Abs. 1; 14 Abs. 1 GG verankerte Verbot der Erdrosselungssteuer gestützt. ${ }^{82}$ Der bereits erwähnte Beschluß des Zweiten Senats zur Vermögensteuer von $1995^{83}$ trägt ganz die Handschrift von Paul Kirchhof. Originelle Produkte seiner Lehre sind besonders der verfassungsrechtliche Schutz des normalen, familiären Gebrauchsvermögens und der Halbteilungsgrundsatz. ${ }^{84}$ Mit dem bereits oben ${ }^{85}$ angesprochenen Halbteilungsgrundsatz weist Paul Kirchhof den Leviathan des Steuerstaates in feste Grenzen und verwirklicht damit den von Geoffrey Brennan und James M. Buchanan in dem berühmten Buch "The Power to Tax" verfolgten Ansatz einer konstitutionellen Begrenzung der Steuerlast. ${ }^{86}$ Paul Kirchhof erweist sich hier als gelehriger Schüler von Klaus Vogel, der seit 1981 Verfassungsgrenzen für Steuern und Staatsaus-

80

81

82

83

84

85

86
P. Kirchhof, Verfassungsrechtliche Rechtfertigung der Steuern (Fn. 78), S. 41.

P. Kirchhof, Verfassungsrechtliche Rechtfertigung der Steuern (Fn. 78), S. 52.

Beschluß vom 25.9.1992 (Fn. 45), S. 169.

Beschluß vom 22.6.1995 (Fn. 56).

Siehe oben S. 533 f. Zu dem Einfluß von Paul Kirchhof auf diese Judikatur siehe Tipke / Lang, Steuerrecht (Fn. 1), § 4 Rz. 214 ff.

Siehe oben S. $533 \mathrm{f}$.

Geoffrey Brennan / James M. Buchanan, The Power to Tax, Analytical Foundations of a Fiscal Constitution, Cambridge University Press 1980. 
gaben fordert. ${ }^{87}$ Fraglich bleibt allerdings, mit welcher Stringenz das Bundesverfassungsgericht künftig den Halbteilungsgrundsatz handhaben wird. Zur Nagelprobe dürfte eine Wiederbelebung der Vermögensteuer werden. Welches Schicksal der Halbteilungsgrundsatz auch erfahren wird, die Notwendigkeit einer freiheitsrechtlichen Rechtfertigung der Steuern gegen einen ausufernden Steuerstaat wird auch nach der Amtszeit von Paul Kirchhof nicht mehr geleugnet werden können.

\section{Bestimmung der Steuergerechtigkeit durch das Sozialstaatsprinzip}

Am Ende dieses Beitrages soll noch kurz über die steuerrechtliche Bedeutung des Sozialstaatsprinzips berichtet werden. Die Bundesrepublik Deutschland ist ebenso wie die Republik Türkei ein sozialer Rechtsstaat (Art. 20 Abs. 1; 28 Abs. 1 GG; Art. 2 TV). Das Sozialstaatsgebot ist im wesentlichen auf den Ausgleich sozialer Ungleichheiten ausgerichtet und gewährleistet zur Sicherung der Menschenwürde (Art. 1 Abs. 1 GG und Präambel der TV) soziale Mindeststandards. ${ }^{88}$ Das bedeutet für das Steuerrecht folgendes:

a) Das Steuerrecht bewirkt den sozialen Ausgleich zunächst durch Fiskalzwecknormen, nämlich durch die konsequente Umsetzung des Leistungsfähigkeitsprinzips. Das Leistungsfähigkeitsprinzip ist auch sozialstaatlich geprägt. Dies zeigt sich besonders in der Lehre vom indisponiblen Einkommen. Analog zur verfassungsrechtlichen Begründung des Anspruchs auf Sozialhilfe gebietet das Sozialstaatsprinzip die bereits behandelte Steuerfreiheit des Existenzminimums in Höhe der Sozialhilfe. ${ }^{89}$

b) Sodann bestimmt das Sozialstaatsprinzip die Umverteilungsgerechtigkeit der Besteuerung durch Sozialzwecknormen. Umverteilungszwecken dient insbesondere der progressive Einkommensteuertarif. Wie bereits dargelegt ${ }^{90}$, qualifizieren wir die Progressionsnorm nicht als Fiskalzwecknorm, sondern als Umverteilungsnorm, die den Sozialzwecknormen zuzuordnen ist. Nach unserer Auffassung erfährt der progressive Einkommensteuertarif seine Rechtfertigung nicht aus dem Gleichheitssatz, sondern aus dem Sozialstaatsprinzip,

87

88

So ständige Rechtsprechung des Bundesverfassungsgerichts, z.B. amtliche Sammlung (BVerfGE), Band 35, S. 355 f. Siehe K. Stern, Das Staatsrecht der Bundesrepublik Deutschland, Band I, 2. Aufl., München 1984, § 21 (Das sozialstaatliche Prinzip); H.F. Zacher, Das soziale Staatsziel, in: Isensee / Kirchhof (Hrsg.), Handbuch des Staatsrechts (Fn. 78), Band I, Heidelberg 1987, § 25 (Das soziale Ziel).

89

90

Siehe S. 528 ff. (Lehre vom "indisponiblen Einkommen"). 
das ein Prinzip der sozialen Gerechtigkeit ist. ${ }^{91}$ Dieses Prinzip rechtfertigt Umverteilung. Die Progression ist Ausfluß umverteilender Sozialstaatlichkeit.

Die Vermögensteuer sowie die Erbschaft- und Schenkungsteuer können als Instrumente zum Abbau des Vermögensgefälles sozialstaatlich gerechtfertigt werden. Die Erbschaftund Schenkungsteuer hat eine Doppelfunktion: Sie ist primär Fiskalzwecksteuer mit dem Ziel, zugewendetes Einkommen zu besteuern. Zusätzlich hat die Erbschaft- und Schenkungsteuer sozialstaatliche Umverteilungsfunktion, die besonders bei hohen Steuersätzen zum Tragen kommt.

\section{Zusammenfassung}

Zusammenfassend ist festzustellen, daß die türkische und die deutsche Verfassung in bezug auf die verfassungsrechtlichen Vorgaben große Ähnlichkeiten aufweisen. Die gleichmäßige Besteuerung nach der Leistungsfähigkeit, der besondere Schutz von Ehe und Familie, die Sozialbindung der Freiheitsrechte und das Sozialstaatsprinzip begründen gemeinsame Vorstellungen von Steuergerechtigkeit. Daher erscheint es als äußerst fruchtbar, die Verwirklichung der Steuergerechtigkeit durch einzelne Normen des Steuerrechts in der türkischen und deutschen Rechtsordnung zu vergleichen. Hierzu will dieser Bericht über die Steuerrechtsdogmatik in Deutschland einen Beitrag leisten.

Dazu K. Tipke, Steuerrechtsordnung (Fn. 1), Band II, S. $406 \mathrm{ff}$. (Das Sozialstaatsprinzip als Komponente der sozialen Gerechtigkeit im Steuerrecht).

Dazu K. Tipke, Steuerrechtsordnung (Fn. 1), Band II, S. 411 ff. (Sozialstaatsprinzip und Steuerprogression). 
collectivized protection approach. The author comes to the conclusion that, generally speaking, the Refugees Act is largely consistent with international conventions and the majority of its provisions satisfy the formal international legal requirements.

\section{Tax Justice in German and Turkish Constitutional Law}

\section{By Joachim Lang}

The article contains a comparative study of those provisions of the German and Turkish constitutions that deal with tax justice. Not surprisingly, both constitutions share the same basic ideas, because the constitution of the Republic of Turkey of 1982 as well as the German constitution have a common ancestor: the French Declaration of Human and Civil Rights of 1789. In particular, both constitutions take up the internationally acknowledged concept of tax justice, the so-called ability-to-pay-principle (i.e. the fair distribution of the tax burden in accordance with each taxpayer's economic ability). On a more specific level, both constitutions utilize similar concepts to define the cornerstones of their respective tax systems: progressive taxation of income, protection against excessive taxation, taxation as a means to finance welfare programs (redistribution of wealth), and preferred tax treatment of married taxpayers and families, which enjoy special constitutional protection. There is, however, one significant difference: While the Turkish constitution sets forth a strict separation of church and state, Germany has always rejected pure laicism. As a result, certain denominations (e.g., the Catholic and the Protestant Church) and some religious communities are granted a special status by the German constitution. 